Pacific Journal of Mathematics

ERGODIC ACTIONS OF PRODUCT GROUPS 


\title{
ERGODIC ACTIONS OF PRODUCT GROUPS
}

\author{
Caroline Series
}

\begin{abstract}
We apply the theory of virtual groups (ergodic groupoids) to the study of commuting groups of transformations.
\end{abstract}

Introduction. Just as a conjugacy class of closed subgroups of a locally compact second countable (l.c.s.c.) group $G$ determines and is determined by a transitive action of $G$, so a virtual subgroup of $G$ determines and is determined by an ergodic action of $G$. The details of this principle are developed by Mackey in [10]. The following approach to questions concerning group actions is indicated: (a) Consider the problem in the case in which the action is transitive and reformulate this as a question concerning closed subgroups of groups. (b) Solve this group theoretic problem. (c) Translate the result back into the language of transitive group actions. (d) Generalise to the properly ergodic case.

Although the theory of virtual groups has been investigated by various authors (Hahn [8], Mackey [10], Ramsay [14], Westman [19]) this procedure has not previously been applied except in the simplest cases [10]. Here we investigate the following problem: consider an ergodic action of the direct product of l.c.s.c. groups $N$ and $H$ on an analytic Borel space $S$ and describe this in terms of actions of $N$ and $H$ separately, together with a suitable twisting. We obtain by the procedure indicated above a description of the required form whenever the $N$ action on $S$ is smooth (i.e. whenever the space of $N$ orbits is analytic). The similarity of the results in the transitive and ergodic cases is seen by comparing Theorems 3.12 and 4.10. The problem arises as a first step in a scheme of reduction of group actions to actions of comparatively simpler groups.

We assume some familiarity with the theory of virtual groups, c.f. [8], [10], [14]. 1 contains preliminary results on group actions. In 2 we introduce the kernel of a homomorphism of groupoids $\pi: S \times H \rightarrow \mathscr{G}, H$ a l.c.s.c. group, $S$ an analytic Borel $H$ space and $\mathscr{G}$ an analytic measured groupoid. 3 deals with steps (a)-(c) of the procedure outlined above, and 4 contains the main result, Theorem 4.10, that the description obtained in 3 is indeed valid, with some slight modifications, in the properly ergodic case. This gives a description of actions of $N \times H$ in the required form whenever the $N$ action on $S$ is smooth.

For basic definitions relating to Borel groups and Borel group actions we refer to [1], [11], [12], [18]. For analytic Borel spaces $X, \mu$; $Y, \nu ; Z, \lambda$ and Borel maps $P: X \rightarrow Y, Q: Z \rightarrow Y$ with $P_{*} \mu \sim \nu \sim Q_{*} \lambda$ 
we define the fibre product $X * Y$ or $X *_{P, O} Y$ and the fibre product measure as in [14] p. 265. $\mathscr{B}(X)$ denotes the Borel sets of $X . \quad M(X, \mu)$ is the measure algebra of $X, \mu$ [14] p. 261. $\nu \cdot g$ is the measure $\nu \cdot g(E)=\nu(E g)$ and $[\nu]$ is the class of the measure $\nu$. We write $\int f d \nu \sim \int g d \mu$ if $\int f d \nu>0 \Leftrightarrow \int g d \mu>0$. Measures are probability measures unless otherwise stated.

The results of this paper formed part of the author's doctoral thesis (Harvard May 1976). She is happy to have the opportunity of acknowledging her indebtedness to her advisor Professor George W. Mackey who suggested the topic of investigation and whose support and guidance gave constant encouragement.

\section{Preliminaries.}

1.1. It is well known that if $G$ is a 1.c.s.c. group and $S$ an analytic Borel $G$ space with quasi-invariant measure $\nu$, then there is a standard Borel space $T$ and a Borel map $P: S \rightarrow T$ such that $P_{*} \nu$ a.a. fibres of $P$ are ergodic $G$ spaces. $T$ is moreover up to Borel isomorphism the unique space with this property. $T$ will be called the standard quotient of $S$ by the $G$ action. We write $S / G$ for the space of $G$ orbits. An action is smooth if $S / G$ is analytic.

We shall make frequent use of the point realisation theorems of Mackey [12] and Ramsay [14], §3. We also recall the uniqueness of the Haar class on a 1.c.s.c. group $G,[18]$ Theorem 8.4, and of the quasiinvariant classes on its quotients by closed subgroups, [18] Theorem 8.19. The natural class on a coset $H g$ of $G$ is the class [ $\nu g]$ where [ $\nu]$ is the Haar class on $H$. In the decomposition

$$
\mu=\int_{\Pi \backslash G} \mu_{s} d p_{*} \mu(s)
$$

of Haar measure $\mu$ on $G$ with respect to projection $p$, the measures $\mu_{s}$ are a.a. in the natural class on $p^{-1}(s),[13]$ p. 104.

1.2. Let $G$ be a l.c.s.c. group and let $S$ be an analytic Borel space with measure $\nu$. Let $T \subseteq S \times G$ be analytic and conull with respect to $\nu x$ Haar. Let $m: T \rightarrow S$ be a Borel map such that

$$
\begin{array}{ll}
m(m(s, g), h)=m(s, g h) & \forall(s, g),(m(s, g), h),(s, g h) \in T \\
m\left(m(s, g), g^{-1}\right)=s & \forall(s, g),\left(m(s, g), g^{-1}\right) \in T .
\end{array}
$$

$m$ is called an almost action of $G$ on $S$.

For a.a. $g \in G,\{s \in S:(s, g) \in T\}$ is conull. Therefore $\nu g$ is de- 
fined a.a. $g \in G$. If $\nu g \sim \nu$ whenever $\nu g$ is defined, then $\nu$ is said to be almost quasi-invariant.

THEOREM 1.3. In the above situation, there exist a standard Borel $G$ space $X$, with quasi-invariant measure $\omega$, and an analytic conull subset $Y \subseteq S$, a Borel isomorphism $J: Y \rightarrow X$, such that $J_{*}(\nu)=\omega$ and

$$
\{(s, g) \in S \times G:(s, g) \in T, s \in Y, s g \in Y, J(m(s, g))=J(s) g\}
$$

is conull in $S \times G$.

Proof. Let $K=\{g \in G:(s, g) \in T$ a.a. $s \in S\} . \quad K$ is conull. Define a map $U: K \rightarrow U\left(L^{2}(S, \nu)\right)$ by

$$
U(k) f(s)=f(m(s, k)) \sqrt{p_{k}(s)}
$$

where $p_{g}$ is the Radon Nikodym derivative of $\nu \cdot g$ with respect to $\nu$.

With respect to the strong operator topology on $U\left(L^{2}(S, \nu)\right), U$ is Borel by [18] Theorem 8.10. By [18] Lemma 8.26 $U$ extends to a Borel homomorphism $G \rightarrow U\left(L^{2}(S, \nu)\right)$. Therefore $M(S, \nu)$ is a Boolean $G$ space. Now apply [12] Theorem 1 and [14] Theorem 3.5.

Definition 1.4. Let $G$ be a 1.c.s.c. group and let $S, S^{\prime}$ be almost analytic Borel $G$ spaces with almost quasi-invariant measures $\nu, \nu^{\prime}$. Let $T: S \rightarrow S^{\prime}$ be a measurable map such that $\{(s, g) \in S \times G: T(m(s, g))=$ $\left.m^{\prime}(T(s), g)\right\}$ is conull in $S \times G$, where $m, m^{\prime}$ define the almost actions in $S, S^{\prime} . \quad T$ is called almost equivariant. If in addition $T$ is a measure theoretic isomorphism, $T$ is called an almost isomorphism.

TheOREM 1.5. Let $H, G$ be 1.c.s.c. groups and let $S$ be an analytic Borel $G$ space with finite quasi-invariant measure $\nu$, and let $j: H \rightarrow G$ be a continuous homomorphism with dense range. Then $s \cdot h=s j(h), s \in S$, $h \in H$, defines a Borel action of $H$ on $S$. If $A \in \mathscr{B}(S)$ is $H$ invariant then there is a $G$ invariant set $B \in \mathscr{B}(S)$ which differs by a null set from $A$. In particular, if $G$ acts ergodically so does $H$, and if in addition $G$ acts freely and $j(H)$ is not closed then $H$ acts properly ergodically.

Proof. That the $H$ action is Borel is clear. Let $A \in \mathscr{B}(S)$ be $H$ invariant. Given $g \in G$, let $h_{n} \in H, j\left(h_{n}\right) \rightarrow g$. Let $x \mapsto U_{x}$ be the representation of $G$ on $U\left(L^{2}(S, \nu)\right)$ defined as in 1.3. By [2] p. 23, $U$ is strongly continuous.

For $x \in G, U_{x} \chi_{A}=\chi_{A \cdot x} \cdot U_{x} 1$ where 1 is the function which is identically 1 on $S$. 
For $h \in H, \chi_{A \cdot h}=\chi_{A}$.

For any $\omega \in L^{2}(S, \nu)$ :

$$
\begin{aligned}
& \left\langle\chi_{A_{g}} U_{g} 1, \omega\right\rangle-\left\langle\chi_{A} U_{g} 1, \omega\right\rangle \\
& =\left\langle U_{g} \chi_{A}, \omega\right\rangle-\left\langle U_{g} 1, \chi_{A} \omega\right\rangle \\
& =\operatorname{Lim}_{n \rightarrow \infty}\left(\left\langle U_{h_{n}} \chi_{A}, \omega\right\rangle-\left\langle U_{h_{n}} 1, \chi_{A} \omega\right\rangle\right) \\
& =\operatorname{Lim}_{n \rightarrow \infty}\left(\left\langle\chi_{A h_{n}} U_{h_{n}} 1, \omega\right\rangle-\left\langle U_{h_{n}} 1, \chi_{A} \omega\right\rangle\right) \\
& =\operatorname{Lim}_{n \rightarrow \infty}\left(\left\langle U_{h_{n}} 1, \chi_{A} \omega\right\rangle-\left\langle U_{h_{n}} 1, \chi_{A} \omega\right\rangle\right) \\
& =0 .
\end{aligned}
$$

Therefore $\chi_{A g} U_{g} 1=\chi_{A} U_{g} 1 . \quad U_{g} 1$ is nonzero a.e. So $(1): \chi_{A g}=\chi_{A}$.

This implies that the Boolean algebra element in $M(S, \nu)$ corresponding to $A$ is invariant under $G$, so by [12] Theorem $3 A$ differs by a null set from a $G$ invariant null set.

It follows easily that if the $G$ action is ergodic, so is that of $H$.

Suppose $\nu$ is ergodic and $G$ acts freely, and suppose the $H$ action is essentially transitive. Let $X \subseteq S$ be the $H$ orbit supporting $\nu$. Pick $g \in G$ such that $X \cap X g=\varnothing$. Then $\nu \cdot g$ is a measure supported on $X g$, and yet $\nu \cdot g \sim \nu$. This is a contradiction, so the $H$ action must be properly ergodic.

2. Kernels of homomorphisms. Although we presume that the reader has some acquaintance with the theory of measured analytic groupoids (virtual groups), we fix notation, to be referred to as the standard notation, by recalling the basic definitions. For further details we refer to [10], [14].

DEFINITION 2.1. An (algebraic) groupoid is a set $\mathscr{G}$ together with a subset $\mathscr{G}^{(2)} \subseteq \mathscr{G} \times \mathscr{G}$ and maps $m: \mathscr{G}^{(2)} \rightarrow \mathscr{G}, \quad m(x, y)=x y ; i: \mathscr{G} \rightarrow \mathscr{G}$, $i(x)=x^{-1}$, with the following properties:

(i) There is a subset $U_{\mathscr{G}}=U \subseteq \mathscr{G}$ of units, so that to each $x \in \mathscr{G}$ there correspond unique left and right units $r(x), d(x)$ respectively, and $(r(x), x),(x, d(x)) \in \mathscr{G}^{(2)} ; r(x) x=x=x d(x)$.

(ii) $(x, y) \in \mathscr{G}^{(2)} \Leftrightarrow d(x)=r(y)$

(iii) $(x, y),(y, z) \in \mathscr{G}^{(2)} \Leftrightarrow(x y, z),(x, y z) \in \mathscr{G}^{(2)}$ and $(x y) z=x(y z)$

(iv) $i^{2}=i d,(i(x), x),(x, i(x)) \in \mathscr{G}^{(2)}, i(x) x=d(x), x i(x)=r(x)$.

Definition 2.2. A measured analytic groupoid $\mathscr{G}, \mu$ is a groupoid $\mathscr{G}$ which is also an analytic Borel space, together with a measure $\mu$ such 
that

(i) $\quad \mathscr{G}^{(2)} \in \mathscr{B}(\mathscr{G} \times \mathscr{G})$

(ii) The maps $m: \mathscr{G}^{(2)} \rightarrow \mathscr{G} ; i: \mathscr{G} \rightarrow \mathscr{G} ; d, r: \mathscr{G} \rightarrow U$ are Borel.

(iii) (Quasi-invariance of $\mu$ ).

(a) $i_{*} \mu \sim \mu$.

Set $F^{s}=r^{-1}(s), s \in U$. For $x \in, \mathscr{G}$, set $T^{x}: F^{d(x)} \rightarrow F^{r(x)}, T^{x}(y)=$ $x y$. Let $\mu=\int_{U} \mu^{s} d r_{*} \mu(s)$ be a decomposition of $\mu$ with respect to $r$, $r_{*} \mu$. Then

(b) There is a $r_{*} \mu$ null set $N \subseteq U$, such that $x \in \mathscr{G}, d(x) \notin N$, $r(x) \notin N \Rightarrow T_{*}^{x} \mu^{d(x)} \sim \mu^{r(x)}$.

2.3. It is always true that $d_{*} \mu \sim r_{*} \mu . \quad \mu$ is symmetric if $d_{*} \mu=$ $r_{*} \mu$. There are always symmetric measures in $[\mu]$, for example $\mu+$ $i_{*} \mu$. We write $\bar{\mu}=r_{*} \mu$.

Set $F_{s}=d^{-1}(s), s \in U$. Let $\mu=\int_{U} \mu_{s} d d_{*} \mu(s)$ be a decomposition of $\mu$ with respect to $d, d_{*} \mu$. For $x \in \mathscr{G}$, set $T_{x}: F_{r(x)} \rightarrow F_{d(x)}, T_{x}(y)=$ $y x$. It follows that there is a null set $N^{\prime} \subseteq U$ such that $x \in \mathscr{G}, d(x) \notin N^{\prime}$, $r(x) \notin N^{\prime} \Rightarrow T_{x} * \mu_{r(x)} \sim \mu_{d(x)}$.

If $U_{0} \subseteq \mathscr{B}(U)$ is conull, then $\left.\mathscr{G}\right|_{U_{0}}=\left\{x \in \mathscr{G}: d(x) \in U_{0}, r(x) \in U_{0}\right\}$ with the restriction of the measure $\mu$ is an analytic measured groupoid called an inessential contraction (i.c.) of $\mathscr{G}$.

Notice $\mathscr{G}^{(2)}=\mathscr{G}_{d, r} * \mathscr{G}$. We always put the fibre product measure $\mu^{(2)}$ on $\mathscr{G}^{(2)}$.

2.4. A groupoid is principal if $x, y \in \mathscr{G}, d(x)=d(y), r(x)=r(y) \Rightarrow$ $x=y$. For any groupoid $\mathscr{G}$, the associated principal groupoid $P(\mathscr{G})$ is $\{(u, v) \in U \times U: u \sim v\} . \quad P: \mathscr{G} \rightarrow P(\mathscr{G}), P(x)=(r(x), d(x))$, induces on $P(\mathscr{G}), P_{*} \mu$ the structure of a measured analytic groupoid ([15] Theorem 6.8). We write $\tilde{\mu}=P_{*} \mu$. Let $\mu=\int_{P(\mathscr{G})} \mu_{u, v} d \tilde{\mu}(u, v)$ be the decomposition of $\mu$ with respect to $P$, and set $\Gamma_{u, v}=F^{u} \cap F_{v}$.

By [15] Theorem 6.8, there is an i.c. of $\mathscr{G}$ on which

2.4 .1

$$
\mu^{u}=\int_{d\left(F^{u}\right)} \mu_{u, v} d d_{*} \mu^{u}(v), \quad \mu_{u}=\int_{r\left(F_{u}\right)} \mu_{v, u} d r_{*} \mu_{u}(v)
$$

$$
\begin{aligned}
& T_{x} * \mu_{t, r(x)} \sim \mu_{t, d(x)}, \quad T_{*}^{x} \mu_{d(x), t} \sim \mu_{r(x), t} \\
& \forall x \in \mathscr{G}, \quad t \sim r(x) \in U .
\end{aligned}
$$

2.4.3 $\int_{U} \int_{d\left(F^{u}\right)} \mu_{u, v} d d_{*} \mu^{u}(v) d \bar{\mu}(u)=\int_{U} \int_{r\left(F_{v}\right)} \mu_{u, v} d r_{*} \mu_{v}(u) d \bar{\mu}(v)$. 
A symmetric measure $\mu$ such that $\mu^{u}, \mu_{u}$ etc. satisfy $2.4 .1-2.4 .3$ on all of $\mathscr{G}$ will be called strictly quasi-invariant.

2.5. For $E \subseteq U$, set $[E]=\{s \in U: \exists \xi \in \mathscr{G}, d(\xi)=s, r(\xi) \in E\}$. [E] is called the saturation of $E$ and is analytic whenever $E$ is. $E \in \mathscr{B}(U)$ is negligible if $[E]$ is null.

Definition 2.6. Let $\mathscr{G}_{i}, \quad \mu_{i} \quad i=1,2$, be measured analytic groupoids. A strict Borel homomorphism $\pi: \mathscr{G}_{1} \rightarrow \mathscr{G}_{2}$ is a Borel map $\pi$ such that

(i) $\quad(x, y) \in \mathscr{G}_{1}^{(2)} \Rightarrow(\pi(x), \pi(y)) \in \mathscr{G}_{2}^{(2)}$ and $\pi(x y)=\pi(x) \pi(y)$.

$\pi$ induces a map $\bar{\pi}: U_{\mathscr{G}_{1}} \rightarrow U_{\mathscr{G}_{2}}$.

(ii) $E \in \mathscr{B}\left(U_{\xi_{2}}\right), E$ negligible $\Rightarrow \bar{\pi}^{-1}(E)$ null.

This definition is relaxed as follows:

A Borel homomorphism $\pi: \mathscr{G}_{1} \rightarrow \mathscr{G}_{2}$ is a Borel map which is a strict homomorphism on some i.c. of $\mathscr{G}_{1}$. ( $\pi$ is not required to be everywhere defined.)

An almost Borel homomorphism $\pi: \mathscr{G}_{1} \rightarrow \mathscr{G}_{2}$ is a Borel map defined a.e. on $\mathscr{G}_{1}$ such that

$$
\left\{(x, y) \in \mathscr{G}_{1}^{(2)}:(\pi(x), \pi(y)) \in \mathscr{G}_{2}^{(2)} \text { and } \pi(x) \pi(y)=\pi(x y)\right\}
$$

is $\mu^{(2)}$ conull in $\mathscr{G}_{1}^{(2)}$.

A homomorphism is normalised if $\bar{\pi}_{*} \bar{\mu}_{1} \sim \bar{\mu}_{2}$ and singular if $\bar{\mu}_{2}(\operatorname{Im} \bar{\pi})=0$. Homomorphisms are frequently called cocycles.

The example which motivated the study of analytic groupoids is of course the following:

Let $G$ be a l.c.s.c. group and $S$ an analytic Borel $G$ space with quasi-invariant measure $\nu$. Then $S \times G, \nu \times$ Haar is an analytic measured groupoid, where

$$
d(s, g)=s g, \quad r(s, g)=s, \quad(s, g)(s g, h)=(s, g h), \quad i(s, g)=\left(s g, g^{-1}\right) .
$$

In particular if $K$ is a closed subgroup of the l.c.s.c. group $G$ there is a correspondence between the groupoid $K \backslash G \times G$ and the group $K$. (C.f. [14] p. 280). Moreover, if $H$ is another l.c.s.c. group there is a correspondence between conjugacy classes of continuous homomorphisms $A: K \rightarrow H$ and similarity classes of strict Borel cocycles $\pi$ : $K \backslash G \times G \rightarrow H$. This is constructed as follows:

Given a homomorphism $A: K \rightarrow H$, let $\alpha: K \backslash G \rightarrow G$ be a Borel section of the projection map with $\alpha(K)=\{e\}$, [3] Lemme 3. Define 
$\pi(A, \alpha): K \backslash G \times G \rightarrow H, \pi(A, \alpha)(s, g)=A\left(\alpha(s) g \alpha(s g)^{-1}\right)$. Up to similarity $\pi(A, \alpha)$ is independent of the choice of $\alpha$; conversely a strict Borel cocycle $\pi: K \backslash G \times G \rightarrow H$ defines a homomorphism $A_{\pi}: K \rightarrow H$ by $A_{\pi}(k)=\pi(K, k) . \quad A_{\pi}$ depends only on the similarity class of $\pi$ and is clearly Borel, hence continuous by [2] p. 23.

In [10] Mackey defined the kernel of certain cocycles $\pi: S \times H \rightarrow G$ (where $S$ is an ergodic $H$ space) as a virtual subgroup of $H$, i.e. as an ergodic $H$ space. This concept can be generalised to the case of an arbitrary normalised Borel cocycle $\pi: \mathscr{G}_{1} \rightarrow \mathscr{G}_{2}$, where $\mathscr{G}_{1}$ are analytic measured groupoids. We shall sketch here only that part of the theory relevant to our problem. For further details we refer to [16].

Let $H$ be a l.c.s.c. group, $S$ an analytic Borel $H$ space with quasi-invariant measure $\nu$, and $\mathscr{G}, \mu$ an analytic measured groupoid. Let $\pi: S \times H \rightarrow \mathscr{G}$ be a normalised Borel cocycle, strict on an i.c. $K$ of $S \times H$. Set $X(\pi)=S *_{\bar{\pi}, r} G$ with fibre product measure $\omega(\pi)$. Define an almost action of $H$ on $X(\pi)$ by

$$
(s, \xi) h=\left(s h, \pi(s, h)^{-1} \xi\right) \quad(s, h) \in K .
$$

Now

$$
\begin{aligned}
\omega(\pi) & =\int_{S} \mu^{\tilde{\pi}(s)} d \nu(s) \\
\omega(\pi) h & =\int_{S} T_{*}^{\pi(s, h)^{-1}} \mu^{\tilde{\pi}(s)} d \nu(s h) \\
& \sim \int_{S} \mu^{\tilde{\pi}(s h)} d \nu(s h) \\
& \sim \int_{S} \mu^{\tilde{\pi}(s)} d \nu(s)
\end{aligned}
$$

whenever $h$ is such that $(s, h) \in K$ a.a. $s \in S$. Therefore $\omega(\pi)$ is almost $H$ quasi-invariant.

This almost action of $H$ is called $\operatorname{Ker} \pi$. Notice that it is not in general ergodic. In fact if we define $D: X(\pi) \rightarrow U, D(s, \xi)=d(\xi)$, we see that $H$ leaves the fibres of $D$ invariant so that $\operatorname{Ker} \pi$ cannot be ergodic unless $\operatorname{Im} \bar{\pi}=\{p t\}$, i.e. unless $\mathscr{G}$ is a group $G$.

According to [10] a cocycle $\pi: S \times H \rightarrow G$ has dense range if Ker $\pi$ is ergodic. More generally, we make the following definitions:

DEFINITION 2.7. $\pi$ has dense range if $D$ is an ergodic decomposition of $\operatorname{Ker} \pi . \quad \pi$ has closed range if $\operatorname{Ker} \pi$ is smooth. $\pi$ is surjective if $\pi$ has dense closed range.

This is motivated as follows: the range closure of a Borel cocycle 
$\pi: S \times H \rightarrow \mathscr{G}$ is defined in [16]. The condition that $D$ be an ergodic decomposition of $\operatorname{Ker} \pi$ is equivalent to requiring that $\mathscr{G}$ be the range closure of $\pi$. In the case in which $\mathscr{G}$ is a group we have the following: (c.f. [10] §7).

Proposition 2.8. Let $H, K, G$ be l.c.s.c. groups and suppose $K$ is a closed subgroup of $H$. Let $A: K \rightarrow G$ be a continuous homomorphism. Then $A$ has dense (resp. closed) range if and only if each associated cocycle $\pi(A, \alpha): K \backslash H \times H \rightarrow G$ has dense (resp. closed) range, where $\alpha: K \backslash H \rightarrow H$ is a Borel section with $\alpha(K)=\{e\}$.

Proof. This is a straightforward calculation using Theorem 1.5, c.f. [17] Propositions IV, 2.1, 2.2.

The kernel we have defined may be thought of as the 'left kernel'. It is sometimes convenient to deal instead with the 'right kernel' defined as follows:

$$
\begin{aligned}
& X^{*}(\pi)=S *_{\bar{\pi}, d} \mathscr{G}, \text { with fibre product measure } \omega^{*}(\pi) \\
& (s, \xi) h=(\operatorname{sh}, \xi \pi(s, h)) \quad(s, \xi) \in X^{*}(\pi), \quad(s, h) \in K
\end{aligned}
$$

Corresponding to $D: X(\pi) \rightarrow U$ we have $R: X^{*}(\pi) \rightarrow U, R(s, \xi)=$ $r(\xi)$. The $H$ equivariant isomorphism $X(\pi) \rightarrow X^{*}(\pi),(s, \xi) \mapsto\left(s, \xi^{-1}\right)$, shows that these constructions are equivalent.

We shall need the following result:

LEMMA 2.9.

$$
\omega(\pi)=\int_{U} \int_{r\left(F_{t}\right)} \lambda_{t^{\prime}} \times \mu_{t^{\prime}, t} d r_{*} \mu_{t}\left(t^{\prime}\right) d \bar{\mu}(t)
$$

and

$$
\omega^{*}(\pi)=\int_{U} \int_{d\left(F^{t}\right)} \lambda_{t^{\prime}} \times \mu_{t^{\prime}, t} d d_{*} \mu^{t}\left(t^{\prime}\right) d \bar{\mu}(t)
$$

are decompositions of $\omega(\pi), \omega^{*}(\pi)$ with respect to $D, R$ respectively, where $\nu=\int_{U} \lambda_{t} d \bar{\mu}(t)$ is the decomposition of $\nu$ with respect to $\bar{\pi}$.

Proof.

$$
\begin{aligned}
& \int_{U} \int_{r\left(F_{t}\right)} \lambda_{t^{\prime}} x \mu_{t^{\prime}, t} d r_{*} \mu_{t}\left(t^{\prime}\right) d \bar{\mu}(t) \\
&=\int_{U} \int_{d\left(F^{t^{\prime}}\right)} \lambda_{t^{\prime}} \times \mu_{t^{\prime}, t} d d_{*} \mu^{t^{\prime}}(t) d \bar{\mu}\left(t^{\prime}\right) \text { by } 2.4 .3
\end{aligned}
$$




$$
\begin{aligned}
& =\int_{U} \lambda_{t^{\prime}} \times \mu^{t^{\prime}} d \bar{\mu}\left(t^{\prime}\right) \\
& =\omega(\pi)
\end{aligned}
$$

and similarly for $\omega^{*}(\pi)$.

2.10. Quasi-equivalence of $G$ spaces. In dealing with nonergodic $G$ spaces (in particular in connection with kernels) a notion weaker than that of isomorphism is useful. Let $S$ be an analytic Borel $G$ space and let $I$ be the unit interval. $S \times I$ becomes a $G$ space if we define $(s, a) g=(s g, a), s \in S, a \in I, g \in G$. Let $S, S^{\prime}$ be analytic Borel $G$ spaces. $S$ is quasi-equivalent (q.e.) to $S^{\prime}$, if there is a $G$ isomorphism $T: S \times I \rightarrow S^{\prime} \times I$ where $S \times I, S^{\prime} \times I$ are $G$ spaces as described above. Roughly speaking, this means that $S$ and $S^{\prime}$ have the same ergodic components as $G$ spaces but not necessarily with the same multiplicities. Ergodic quasi-equivalent $G$ spaces are necessarily $G$ isomorphic. This corresponds to the notion of quasi-equivalence for von-Neumann algebras. The application of quasi-equivalence in the context of kernels of cocycles is indicated by the following result, which we shall not prove here.

THEOREM 2.11. Let $\pi: S \times H \rightarrow \mathscr{G}$ be a normalised Borel cocycle and let $A: \mathscr{G} \rightarrow \mathscr{G}^{\prime}$ be a normalised cocycle which is also a similarity. Then $\operatorname{Ker} \pi$ is q.e. to $\operatorname{Ker} A \pi$.

REMARK 2.12. Let $Z$ be an analytic Borel space with measure $\nu$. Up to a null set, $Z$ is Borel isomorphic to one of the following types:

(1) $J_{n}$, an atomic space with $n$ atoms, $n \in Z^{+} \cup\{0\}$

(2) $J_{0}=I$, the unit interval with Lebesgue measure

(3) $J_{-n}=I \cup J_{n}, n \in Z^{+} \cup\{0\}$.

We use $J$ to denote any of these types $J_{n}$.

Now suppose that $S, S^{\prime}$ are $G$ spaces and that $S \times J$ is $G$ isomorphic to $S^{\prime}$. Then since $J \times I \cong I$, we have $S \times I \cong S^{\prime} \times I$ and hence $S, S^{\prime}$ are q.e. $G$ spaces.

The following known result ([17] Proposition B.6) is useful in this context:

Proposition 2.13. Let $X$ be an analytic Borel space with measure $\mu$, up to a null set isomorphic to $I$. Let $Y$ be an analytic Borel space and let $p: X \rightarrow Y$ be measurable. Let $\mu=\int_{Y} \mu_{y} d p_{*}(\mu)(y)$ be a decomposition of $\mu$ with respect to $p$. Suppose that for a.a. $y$ the $\mu_{y}$ are of the same type $J$. Then there is a measure theoretic isomorphism $T: X \rightarrow Y \times J$ with $p=q T$ a.e., where $q: Y \times J \rightarrow Y$ is projection. 
3. Transitive actions of product groups. In this section we take up the original problem and investigate (a)-(c) of the introduction in turn. Recall that our aim is to describe ergodic actions of a product group $N \times H$ in terms of $N$ and $H$ separately. The reformulation of this problem required in (a) is clear: since transitive actions of $N \times H$ correspond to closed subgroups $K \subseteq N \times N$ we seek to describe such subgroups in terms of closed subgroups (transitive actions) of $N$ and $H$ separately. The algebraic solution of (b) is simple: the 'twisting' referred to in the introduction is in this case a homomorphism. The topological problem is complicated by the possibility that the projection of $\mathrm{K}$ on $\mathrm{H}, \mathrm{K}_{2}$, may not be closed. We give a complete solution of the problem only under the assumption that $K_{2}$ is closed. Finally we take up (c) and reformulate our results in terms of transitive actions. The final description, which will be generalised to the ergodic case in 4 is contained in Theorem 3.12.

We begin by giving an algebraic description of subgroups of a direct product. This description is due to Mackey; as far as we are aware it does not appear in the literature.

THEOREM 3.1. Let $N, H$ be groups. Let $K_{1}, L_{1}$ be subgroups of $N$ with $L_{1}$ normal in $K_{1}$; and let $K_{2}, L_{2}$ be subgroups of $H$ with $L_{2}$ normal in $K_{2}$. Suppose $\phi: K_{2} / L_{2} \rightarrow K_{1} / L_{1}$ is an isomorphism. Let $p_{i}: K_{i} \rightarrow K_{i} / L_{i}$ denote the projection maps. Then

$$
\Lambda\left(K_{1}, K_{2}, \phi\right)=\left\{(n, h) \in N \times H: \phi p_{2}(h)=p_{1}(n)\right\}
$$

is a subgroup of $N \times H$. Moreover all subgroups of $N \times H$ are obtained in this manner.

Proof. We leave this as an exercise for the reader.

We turn to the topological part of the problem, namely the characterization of closed subgroups of a direct product of l.c.s.c. groups. A partial answer is given by

THEOREM 3.2. Let $N, H$ be l.c.s.c. groups and let $K \subseteq N \times H$ be a closed subgroup. Define $K_{i}, L_{i}, \phi$ as in Theorem 3.1. Then $L_{1}, L_{2}$ are closed in $N, H$ and $K_{1}, K_{2}$ can be given l.c.s.c. topologies so that the injections $i_{1}: K_{1} \rightarrow N, i_{2}: K_{2} \rightarrow H$ are continuous and $\phi$ is a topological isomorphism. If, for example, $K_{1}$ is closed, this topology coincides with the one induced by $N$.

Proof. It is clear that $L_{1}, L_{2}$ are closed in $N, H . \quad K / L_{1}$ is a l.c.s.c. group and 


$$
K_{2} \rightarrow K / L_{1}, \quad h \mapsto\left(\phi\left(p_{2}(h)\right), h\right)
$$

is an isomorphism. This induces a l.c.s.c. topology on $K_{2}$.

Similarly, $K_{1} \rightarrow K / L_{2}, \quad n \mapsto\left(n, \phi^{-1}\left(p_{1}(n)\right)\right)$ is an isomorphism and induces a l.c.s.c. topology on $K_{1}$.

With these topologies $i_{1}, i_{2}$ are clearly continuous. If $K_{1}$ is closed then $i_{1}$ is a continuous homomorphism onto $K_{1}$ topologized as a subspace of $N$, hence by the closed graph theorem [9] p. 213 a homeomorphism.

To show that $\phi$ is a homeomorphism it is sufficient by the closed graph theorem to show that $\phi$ has a closed graph. Since $i_{1} \times i_{2}: K_{1} \times K_{2}$ $\rightarrow \mathrm{N} \times H$ is continuous, $K$ is closed in $K_{1} \times K_{2} . \quad K$ is saturated with respect to $L_{1} \times L_{2}$, therefore the image of $K$ in $K_{1} \times K_{2} / L_{1} \times L_{2}$ is closed. This image (with the order of factors reversed) is the graph of $\phi$.

We would like to know the circumstances under which the subgroups $\Lambda\left(K_{1}, K_{2}, \phi\right)$ of Theorem 3.1 are closed. One possibility is that at least one of $K_{1}, K_{2}$ is closed:

THEOREM 3.3. Let $N, H$ be l.c.s.c. groups. Let $L_{1}$ be a closed subgroup of $N$ and let $K_{2}, L_{2}$ be closed subgroups of $H$. Let $K_{1}$ be a subgroup of $N$ which has a l.c.s.c. topology so that $i_{1}: K_{1} \rightarrow N$ is continuous. Suppose $L_{i}$ is normal in $K_{i}$. Let $\phi: K_{2} / L_{2} \rightarrow K_{1} / L_{1}$ be a topological isomorphism. Then $\Lambda\left(K_{1}, K_{2}, \phi\right)$ is a closed subgroup of $N \times H$.

Proof. By Theorem 3.1, $\Lambda\left(K_{1}, K_{2}, \phi\right)$ is a subgroup of $N \times H$.

Let $\bar{K}_{1}$ be the closure of $K_{1}$ in $N$. Regard $\phi$ as a continuous homomorphism $K_{2} / L_{2} \rightarrow \bar{K}_{1} / L_{1}$. The graph of $\phi$,

$$
G(\phi)=\left\{(u, \phi(u)) \in K_{2} / L_{2} \times \bar{K}_{1} / L_{1}\right\}
$$

is closed. $\left(p_{2} \times p_{1}\right)^{-1}(G(\phi))$ is closed in $K_{2} \times \bar{K}_{1}$.

Therefore $\left(p_{2} \times p_{1}\right)^{-1}(G(\phi))$ is closed in $H \times N$. On reversing factors we have precisely the set $\Lambda\left(K_{1}, K_{2}, \phi\right)$.

If $N$ is compact, this result is complete:

Proposition 3.4. Let $N \times H$ be a direct product of l.c.s.c. groups and suppose that $N$ is compact. Let $K \subseteq N \times H$ be a closed subgroup. Then $\mathrm{K}_{2}$, the projection of $\mathrm{K}$ on $\mathrm{H}$, is closed.

Proof. This is a standard compactness argument.

When neither $K_{1}$ nor $K_{2}$ is closed in $N, H$ the subgroup $\Lambda\left(K_{1}, K_{2}, \phi\right)$ 
of $N \times H$ may or may not be closed:

ExAmple 3.5. $N=H=\mathbf{R}, K_{1}=K_{2}=Q, L_{1}=L_{2}=\{0\}, \phi=i d$.

$\Lambda\left(K_{1}, K_{2}, \phi\right)$ is not closed.

ExAmple 3.6. $N=H=\mathbf{R}, \beta \in \mathbf{R}$ irrational.

$$
K=\{(n \beta+m, n \beta+n+m) \in \mathbf{R} \times \mathbf{R}: n, m \in \mathbf{Z}\}
$$

$K$ is closed but neither $K_{1}$ nor $K_{2}$ is closed.

In the case in which $K_{2}$ is closed, Theorems 3.2 and 3.3 give a complete solution of (b) of the introduction, that is a description of all possible $K \subseteq N \times H$ in terms of subgroups of $N$ and $H$ and a homomorphism between them. We now turn to (c) and reformulate this description in terms of group actions.

Definition 3.7. A closed subgroup of a product of l.c.s.c. groups $N$ and $H$ is reduced if $\bar{K}_{1}=N, \bar{K}_{2}=H$. A reduced subgroup $K \subseteq N \times H$ is representable if there are a l.c.s.c. group $G$, and continuous homomorphisms $A_{1}: N \rightarrow G, A_{2}: H \rightarrow G$ with dense range so that the $N \times H$ action on $G$ given by $g \cdot(n, h)=A_{2}(h)^{-1} g A_{1}(n)$ is transitive with Stab $\{e\}=$ $K$. More generally a subgroup $K$ is representable if it is representable regarded as a subgroup of $\bar{K}_{1} \times \bar{K}_{2}$.

Now with the notation of Theorem 3.2 with $K_{2}$ closed, set $G=$ $\bar{K}_{1} / L_{1}$. As in Theorem 3.2 we have a continuous map $\phi_{2}: \bar{H}_{2} \rightarrow G$. Let $\phi_{1}: \bar{K}_{1} \rightarrow G$ be projection. Consider the action of $\bar{K}_{1} \times \bar{K}_{2}$ on $G$ given by $g \cdot(n, h)=\phi_{2}(h)^{-1} g \phi_{1}(n), g \in G,(n, h) \in \bar{K}_{1} \times \bar{K}_{2}$. This is clearly transitive and Stab $\{e\}=\Lambda\left(K_{1}, K_{2}, \phi\right)=K$. Therefore $K$ is representable. Notice that $\operatorname{Ker} \phi_{1}=L_{1}, \operatorname{Ker} \phi_{2}=L_{2}$.

We next show that representable subgroups are defined by $N \times H$ actions of a special form which we call models.

Definition 3.8. Let $N, H, G$ be l.c.s.c. groups. Let $S_{1}, S_{2}$ be standard $N, H$ spaces with quasi-invariant measures $\nu_{1}, \nu_{2}$ respectively. Let $\pi_{1}: S_{1} \times N \rightarrow G, \pi_{2}: S_{2} \times K \rightarrow G$ be strict Borel cocycles with dense range. Define an $N \times H$ action on $\Sigma=$ $\Sigma\left(S_{1}, \pi_{1}, S_{2}, \pi_{2}, G\right)=S_{1} \times S_{2} \times G$ :

$$
\begin{aligned}
& (x, y, g) n=\left(x n, y, g \pi_{1}(x, n)\right) \quad(x, y, g) \in \Sigma, \quad n \in N \\
& (x, y, g) h=\left(x, y h, \pi_{2}(y, h)^{-1} g\right) \quad h \in H \text {. }
\end{aligned}
$$

An $N \times H$ action of this form will be called a model and be denoted by $M=M\left(S_{1}, \pi_{1}, S_{2}, \pi_{2}, G\right)$. We always put the measure class $\left[\nu_{1} \times \nu_{2} \times\right.$ 
Haar] on $\Sigma$. It is clear that this class is invariant under the $N \times H$ action.

The next result shows that representable subgroups give rise to model actions.

THEOREM 3.9. Let $K \subseteq N \times H$ be a representable closed subgroup of the product of l.c.s.c. groups $N, H$ defined by continuous homomorphisms $A_{i}: \bar{K}_{t} \rightarrow G$ for some l.c.s.c. group $G$. Let $\alpha_{1}: \bar{K}_{1} \backslash N \rightarrow N, \alpha_{2}: \bar{K}_{2} \backslash H \rightarrow H$ be Borel sections of the projection maps with $\alpha_{i}\left(\bar{K}_{i}\right)=e$. Set $\pi_{i}=$ $\pi\left(A_{i}, \alpha_{\imath}\right)$ as in 2. Then $M\left(\bar{K}_{1} \backslash N, \pi_{1}, \bar{K}_{2} \backslash H, \pi_{2}, G\right)$ is a transitive action of $N \times H$ with $\operatorname{Stab}\left\{\bar{K}_{1}, \bar{K}_{2}, e\right\}=K$.

Proof. This is a routine check from the definitions.

To pass to the ergodic case, we need to have a description of $\bar{K}_{t}$ which generalises to group actions. This is given by

Proposition 3.10. Let $N, H$ be l.c.s.c. groups and let $S$ be a standard transitive $N \times H$ space defined by the closed subgroup $K$. Then the standard quotients of $S$ by the $N$ and $H$ actions are $\bar{K}_{2} \backslash H, \bar{K}_{1} \backslash N$ respectively.

Proof. We prove the result for the $N$ action.

We have a measure theoretic isomorphism $K \backslash N \times H \cong$ $K \backslash N \times \bar{K}_{2} \times \bar{K}_{2} \backslash H$, where we have the natural measure class on each factor. The projection $P: K \backslash N \times H \rightarrow \bar{K}_{2} \backslash H$ is $N$-equivariant. The $N$ invariant sets in $K \backslash N \times \bar{K}_{2}$ are precisely the $K_{2}$ invariant sets in $\bar{K}_{2}$, so that by Theorem 1.3 they are either null or conull. Hence $P$ is an ergodic decomposition of the $N$ action.

We can now state our results in a form which can be immediately generalised to the ergodic case.

Proposition 3.11. Let $K \subseteq N \times H$ be a closed subgroup of the product of l.c.s.c. groups. $K$ is representable whenever $K \backslash N \times H / N$ is analytic, hence in particular whenever $N$ is compact.

Proof. Immediate from 3.4, 3.8, 3.10.

THEOREM 3.12. Let $N, H$ be l.c.s.c. groups and let $K \subseteq N \times H$ be a representable closed subgroup of their product. Then there exist a transitive standard Borel $\mathrm{N}$ space $\mathrm{S}_{1}$, a transitive standard Borel $\mathrm{H}$ space $\mathrm{S}_{2}$, with quasi-invariant measures $\nu_{1}, \nu_{2}$, a l.c.s.c. group $G$ and Borel cocycles $\pi_{1}: S_{1} \times N \rightarrow G, \pi_{2}: S_{2} \times H \rightarrow G$, so that $M\left(S_{1}, \pi_{1}, S_{2}, \pi_{2}, G\right)$ is the transi- 
tive action of $N \times H$ determined by $K . \quad S_{i}, \pi_{i}, G$ have the following properties:

(i) $S_{1}, \nu_{1}$ and $S_{2}, \nu_{2}$ are standard quotients of $S=K \backslash N \times H$ by the $H$ and $N$ actions respectively.

(ii) $\pi_{1}, \pi_{2}$ have dense range.

(iii) $\pi_{1}$ (resp. $\pi_{2}$ ) is surjective $\Leftrightarrow S / N$ (resp. $S / H$ ) is standard.

(iv) $\operatorname{Ker} \pi_{1}$ (resp. Ker $\pi_{2}$ ) is q.e. to the $N$ (resp. H) action on $S$.

Proof. The isomorphism of the $N \times H$ action on $S$ with $M\left(S_{1}, \pi_{1}, S_{2}, \pi_{2}, G\right)$ follows from Theorem 3.9, setting $S_{1}=\bar{H}_{1} \backslash N, S_{2}=$ $\bar{K}_{2} \backslash H$. The equivalence of measures follows from 1.1. (i) follows from Proposition 3.10. (ii) follows from the definitions of $\pi_{i}$ given in Theorem 3.9 and Proposition 2.8. (iii) By Proposition 2.8 and the definition of $\pi_{1}, \pi_{1}$ is surjective $\Leftrightarrow A_{1}$ is surjective $\Leftrightarrow K_{2}$ is closed. As in 2.10, $S / N=K_{2} \backslash H$, and the result for $\pi_{1}$ follows. The proof for $\pi_{2}$ is similar. (iv) follows immediately from the definitions of $\operatorname{Ker} \pi_{1}$ and of quasi-equivalence, and the isomorphism of the $N \times H$ action on $S$ with $M\left(S_{1}, \pi_{1}, S_{2}, \pi_{2}, G\right)$.

REMARK 3.13. Notice that the condition that $K_{2}$ be closed is not necessary for $K$ to be representable. In fact it is not hard to show that $K$ is representable whenever it is normal in $\bar{K}_{1} \times \bar{K}_{2}$.

4. Ergodic actions of $N \times H$. The results of 3.11 and 3.12 were presented in such a way as to make sense in the properly ergodic case. Indeed, if we merely replace the word 'transitive' by 'ergodic' the results still have content. In this section we investigate (d) of the introduction, namely to what extent these results remain true in the ergodic case. Suppose $N \times H$ is the direct product of the l.c.s.c. groups $N$ and $H$ and $S$ is an analytic Borel $N \times H$ space with quasi-invariant ergodic measure $\nu$. Guided by Proposition 3.11 we shall look for results in the case in which the $N$ action is smooth. The following modifications are necessary in Theorem 3.12: $G$ is replaced by an analytic measured groupoid $\mathscr{G}$ and $\Sigma=S_{1} \times S_{2} \times G$ is replaced by a suitable fibred product $S_{1} * S_{2} * \mathscr{G}$.

We begin with a detailed description of the class of ergodic actions of $N \times H$ which we will call models. Throughout $N$ and $H$ are l.c.s.c. groups and $S_{1}, S_{2}$ are analytic Borel $N$ and $H$ spaces respectively, with quasi-invariant ergodic measures $\nu_{1}, \nu_{2} . \mathscr{G}, \mu$ is a measured analytic groupoid, $\mu$ symmetric, and $\pi_{1}: S_{1} \times N \rightarrow \mathscr{G}, \pi_{2}: S_{2} \times H \rightarrow \mathscr{G}$ are normalized Borel cocycles with dense range. We use the standard notation of 2 for $\mathscr{G}, \mu$.

$X\left(\pi_{2}\right)=S_{1} *_{\pi_{2}, r} \mathscr{G}$ with fibre product measure $\omega\left(\pi_{2}\right)$. 
Define $D: X\left(\pi_{2}\right) \rightarrow U_{\xi}, D(s, \xi)=d(\xi)$ as in 2 .

By [14] p. 265, $D_{*}\left(\omega\left(\pi_{2}\right)\right)=\bar{\mu}$.

Set $\Sigma\left(S_{1}, \pi_{1}, S_{2}, \pi_{2}, \mathscr{G}\right)=S_{1} *_{i_{1}, D} X\left(\pi_{2}\right)$ with the fibre product measure $m=m\left(S_{1}, \pi_{1}, S_{2}, \pi_{2}, \mathscr{G}\right)$.

LEMma 4.1. $\Sigma\left(S_{1}, \pi_{1}, S_{2}, \pi_{2}, \mathscr{G}\right)$ is in a natural way an analytic Borel $H$ space and $m$ is quasi-invariant, whenever $\pi_{1}, \pi_{2}$ are strict.

Proof. $\quad H$ acts on $X\left(\pi_{2}\right)$ preserving $\omega\left(\pi_{2}\right)$ as in 2. Define an action of $H$ on $\Sigma$ by $(s, w) h=(s, w h) w \in X\left(\pi_{2}\right), s \in S_{1}, h \in H$. This action clearly makes $\Sigma$ an analytic Borel $H$ space.

To see that $m$ is quasi-invariant it is sufficient to see that if

$$
\omega\left(\pi_{2}\right)=\int_{U} \omega_{u} d \bar{\mu}(u)
$$

is the decomposition of $\omega\left(\pi_{2}\right)$ with respect to $D, \bar{\mu}$, then $\omega_{u} \cdot h \sim \omega_{u}$ a.a. $u \in U$. This follows since $D^{-1}(u) h \subseteq D^{-1}(u)$ and $\omega\left(\pi_{2}\right) \cdot h \sim \omega\left(\pi_{2}\right)$.

LEMMA 4.2. Whenever $\pi_{1}, \pi_{2}$ are strict, $\Sigma$ is an analytic Borel $N$ space and $m$ is $N$ quasi-invariant, moreover the actions of $N$ and $H$ commute.

Proof. Our method is to exhibit $\Sigma$ in a more symmetrical form:

(a) Alternative definition of $\Sigma$ :

Clearly

$$
\Sigma=\left\{\left(s_{1}, s_{2}, \xi\right) \in S_{1} \times S_{2} \times \mathscr{G}: \bar{\pi}_{1}\left(s_{1}\right)=d(\xi), \bar{\pi}_{2}\left(s_{2}\right)=r(\xi)\right\}
$$

Let $\nu_{\imath}=\int_{u} \nu_{u}^{\imath} d \bar{\mu}(u), i=1,2$, be decompositions of $\nu_{\imath}$ with respect to $\bar{\pi}_{l}$, $\bar{\mu}$. Let $P: \mathscr{G} \rightarrow P(\mathscr{G}), \xi \mapsto(r(\xi), d(\xi))$ be the map of $\mathscr{G}$ onto its associated principal groupoid $P(\mathscr{G})$. (cf. 2.4). Let $\mu=\int_{P(\mathscr{G})} \mu_{u, v} d \tilde{\mu}(u, v)$ be a decomposition of $\mu$ with respect to $P, P_{*} \mu=\tilde{\mu}$. $\quad \mu_{u, v}$ is a measure on $\Gamma_{u, v}=P^{-1}((u, v))$.

For $u, v \in U$ with $u \sim v$ :

$$
\nu_{v}^{1} \times \nu_{u}^{2} \times \mu_{u, v} \text { is a measure on } \bar{\pi}_{1}^{-1}(v) \times \bar{\pi}_{2}^{-1}(u) \times \Gamma_{u, v} .
$$

For $A_{i} \in \mathscr{B}\left(S_{1}\right), C \in \mathscr{B}(\mathscr{G})$, the function

$$
(u, v) \mapsto \nu_{v}^{1}\left(A_{1}\right) \nu_{u}^{2}\left(A_{2}\right) \mu_{u, v}(C)
$$


is measurable on $P(\mathscr{G})$ with support contained in $\Sigma$. Therefore $\tilde{m}=$ $\int_{P(\mathscr{G})} \nu_{v}^{1} \times \nu_{u}^{2} \times \mu_{u, v} d \tilde{\mu}(u, v)$ is a measure on $\Sigma$.

(b) $\tilde{m}=m$ :

The fibering of $\omega\left(\pi_{2}\right)$ with respect to $D, \bar{\mu}$ is

$$
\omega\left(\pi_{2}\right)=\int_{U} \int_{r\left(F_{v}\right)} \nu_{u}^{2} \times \mu_{u, v} d r_{*}\left(\mu_{v}\right)(u) d \bar{\mu}(v) \text { by Lemma 2.9. }
$$

Therefore

$$
\begin{aligned}
m & =\int_{U} \nu_{v}^{1} \times\left(\int_{r\left(F_{v}\right)} \nu_{u}^{2} \times \mu_{u, v} d r_{*}\left(\mu_{v}\right)(u)\right) d \bar{\mu}(v) \\
& =\int_{U} \nu_{v}^{1} \times \nu_{u}^{2} \times \mu_{u, v} d \tilde{\mu}(u, v) \quad \text { by } 2.4 \\
& =\tilde{m}
\end{aligned}
$$

(c) $\Sigma=X^{*}\left(\pi_{1}\right) *_{R, \bar{\pi}_{2}} S_{2}$ (with the order of factors altered).

This follows by symmetry, recalling that

$$
X^{*}\left(\pi_{1}\right)=S_{1} *_{\pi_{1}, d} \mathscr{G} \quad \text { and } \quad R(s, \xi)=r(\xi) \quad s \in S_{1}, \quad \xi \in \mathscr{G}
$$

There is a natural action of $N$ on $X^{*}\left(\pi_{1}\right)$ which extends to an $N$ action on $\Sigma=X^{*}\left(\pi_{1}\right) * S_{2}$ preserving [ $m$ ] exactly as the $H$ action on $X\left(\pi_{2}\right)$ is extended in Lemma 4.1. It is clear that the $N$ and $H$ actions commute.

Definition 4.3. $M=M\left(S_{1}, \pi_{1}, S_{2}, \pi_{2}, \mathscr{G}\right)$ is the $N \times H$ action on $\Sigma\left(S_{1}, \pi_{1}, S_{2}, \pi_{2}, \mathscr{G}\right)$ defined in Lemmas 4.1, 4.2 above, namely:

$$
\begin{aligned}
& \left(s_{1}, s_{2}, \xi\right) n=\left(s_{1} n, s_{2}, \xi \pi_{1}\left(s_{1}, n\right)\right) \quad s_{i} \in S_{i}, \xi \in \mathscr{G}, h \in H, n \in N \\
& \left(s_{1}, s_{2}, \xi\right) h=\left(s_{1}, s_{2} h, \pi_{2}\left(s_{2}, h\right)^{-1} \xi\right) .
\end{aligned}
$$

An action of this kind will be called a model.

Notice that in the case $\mathscr{G}=G$, a l.c.s.c. group, these actions reduce to the models of 3.8 .

If $\pi_{1}, \pi_{2}$ are only almost cocycles, then the maps $\bar{\pi}_{1}, \bar{\pi}_{2}$ are defined a.e. $S_{1}, S_{2}$, so that the fibre product $S_{1} * S_{2} * \mathscr{G}$ may still be defined.

Lemma 4.4. If in Definition $4.3, \pi_{1}$ and $\pi_{2}$ are replaced by almost Borel cocycles, then $M\left(S_{1}, \pi_{1}, S_{2}, \pi_{2}, \mathscr{G}\right)$ is an almost $N \times H$ space.

Proof. By [14] Theorem 5.1, we may alter $\pi_{1}$ and $\pi_{2}$ on null sets and 
find conull sets $E_{1} \in \mathscr{B}\left(S_{1} \times N\right), E_{2} \in \mathscr{B}\left(S_{2} \times H\right)$, on which $\pi_{1}, \pi_{2}$ are strict.

$$
\begin{array}{ll}
\text { 4.4.1 } & \left(s_{1}, s_{2}, \xi\right) n \quad \text { is defined whenever } \quad\left(s_{1}, n\right) \in E_{1} . \\
\text { 4.4.2 } & \left(s_{1}, s_{2}, \xi\right) h \quad \text { is defined whenever } \quad\left(s_{2}, h\right) \in E_{2} .
\end{array}
$$

It is clear that

$$
\left\{\left(s_{1}, s_{2}, \xi, n, h\right) \in \Sigma \times N \times H:\left(s_{1}, n\right) \in E_{1},\left(s_{2}, h\right) \in E_{2}\right\} \text { is conull in } \Sigma \text {. }
$$

It is easy to check as in Lemmas 4.1, 4.2 that 4.4.1, 4.4.2 define an almost action of $N \times H$ on $\Sigma$ and that $m$ is almost quasi-invariant.

If $\pi_{1}, \pi_{2}$ are not strict, Lemma 4.4 and 1.1 enable us to find a standard Borel space $\tilde{\Sigma}$ and an action $\tilde{M}$ of $N \times H$ on $\tilde{\Sigma}$, almost isomorphic to the almost $N \times H$ action of 2.4 on $\Sigma$. Such an action will again be called a model.

Lemmas $4.5-4.8$ below are proved under the assumption that $\pi_{1}, \pi_{2}$ are strict Borel cocycles. The results are equally valid, mutatis mutandi, for the almost action $M\left(S_{1}, \pi_{1}, S_{2}, \pi_{2}, \mathscr{G}\right)$ obtained if $\pi_{1}, \pi_{2}$ are not strict or the corresponding action $\tilde{M}$.

LEMMA 4.5. $S_{1}, \nu_{1}$ and $S_{2}, \nu_{2}$ are analytic quotients of $\Sigma$ by the $H$ and $N$ actions respectively.

Proof. We prove this for $S_{1}, \nu_{1}$.

Since $\pi_{2}$ has dense range, $D$ is an ergodic decomposition of $X\left(\pi_{2}\right)$. Let $\omega\left(\pi_{2}\right)=\int_{U} \omega_{u} d \bar{\mu}(u)$ be the decomposition of $\omega\left(\pi_{2}\right)$ with respect to $D, \bar{\mu}$. For a.a. $u \in U, D^{-1}(u), \omega_{u}$ is an ergodic $H$ space.

Let $q_{1}: \Sigma \rightarrow S_{1}$ be projection and let $m=\int_{S_{1}} m_{t} d \nu_{1}(t)$ be a decomposition of $m$ with respect to $q_{1}, \nu_{1}$. By [14] p. 266, for a.a. $t \in S_{1}, q_{1}^{-1}(t)$, $m_{t}$ is isomorphic to $D^{-1}\left(\bar{\pi}_{1}(t)\right) \times\{t\}, \omega_{\bar{\pi}_{1}(t)}$

Therefore $q_{1}$ is an ergodic decomposition of the $H$ action on $\Sigma$.

COROLlary 4.6. $\Sigma$ is an ergodic $N \times H$ space.

Proof. By assumption $N$ acts ergodically on $S_{1}$. This together with 4.5 gives the result.

Lemma 4.7. $\Sigma / N(\operatorname{resp} . \Sigma / H)$ is analytic $\Leftrightarrow \pi_{1}$ (resp. $\pi_{2}$ ) has closed range. 
Proof. $\Sigma / N=X^{*}\left(\pi_{1}\right) / N * S_{2}$.

Therefore

$\Sigma / N$ is analytic $\Leftrightarrow X^{*}\left(\pi_{1}\right) / N$ is analytic $\Leftrightarrow \pi_{1}$ has closed range.

LEMmA 4.8. $\operatorname{Ker} \pi_{1}\left(\operatorname{resp} . \operatorname{Ker} \pi_{2}\right)$ is quasi-equivalent to the action of $N$ (resp. $H$ ) on $\Sigma$.

Proof. This follows immediately from the following general result:

Proposition 4.9. Let $X, Y, Z$ be analytic Borel spaces wiih probability measures $\lambda, \mu, \nu$ respectively. Let $p: X \rightarrow Z, q: Y \rightarrow Z$ be Borel maps such that $p_{*}(\lambda) \sim \nu \sim q_{*}(\mu)$. Suppose there is a Borel action of the l.c.s.c. group $G$ on $X$ such that $p^{-1}(z) G \subseteq p^{-1}(z) \forall z \in Z$, and $\lambda$ is quasi-invariant. Then $(x, y) g=(x g, y)$ defines a Borel action of $G$ on $F=X *_{Z} Y$ so that the fibre product measure $\lambda * \mu$ is quasi-invariant, and this action is quasi-equivalent to the action of $G$ on $X$.

Proof. That $(x, y) g=(x g, y)$ defines a Borel action is clear; that $\lambda * \mu$ is quasi-invariant follows as in the proof of Lemma 4.1.

By $2.12 q^{-1}(z) \cong J_{n}$ for some $n \in N, \quad \forall z \in Z$. Let $Z_{n}=$ $\left\{z \in Z: q^{-1}(z) \cong J_{n}\right\}, X_{n}=p^{-1}\left(Z_{n}\right), Y_{n}=q^{-1}\left(Z_{n}\right), F_{n}=X_{n} *_{Z_{n}} Y_{n}$.

$F=\cup F_{n}$ and each $F_{n}$ is $G$ invariant. It is therefore sufficient to show that $F_{n} \times I \cong X_{n} \times I \forall n$, where $F_{n} \times I, X_{n} \times I$ are $G$ spaces with trivial actions on the second factors. By 2.13 there is an isomorphism $T_{n}: Y_{n} \rightarrow Z_{n} \times J_{n} \quad$ and $p T_{n}=q$ where $p: Z_{n} \times J_{n} \rightarrow Z_{n} \quad$ is projection. Therefore $F_{n} \cong X_{n} \times J_{n}$, with trivial $G$ action on $F_{n}$. This gives the result.

We are now able to state our generalisation of 3.11 and 3.12.

THEOREM 4.10. Let $N, H$ be l.c.s.c. groups and let $S$ be an analytic Borel $N \times H$ space with quasi-invariant measure $\nu$. Suppose that $S / N$ is analytic. Then there exist an analytic Borel $N$ space $S_{1}$ and an analytic Borel $\mathrm{H}$ space $S_{2}$ with quasi-invariant ergodic measures $\nu_{1}, \nu_{2}$; an analytic measured groupoid $\mathscr{G}, \mu$; and normalised Borel cocycles $\pi_{1}$ : $S_{1} \times N \rightarrow \mathscr{G}, \pi_{2}: S_{2} \times H \rightarrow \mathscr{G}$, such that $\tilde{M}\left(S_{1}, \pi_{1}, S_{2}, \pi_{2}, \mathscr{G}\right)$ is isomorphic to the action of $N \times H$ on $S . S_{l}, \pi_{i}, \mathscr{G}$ have the properties:

(i) $S_{1}, \nu_{1}$ and $S_{2}, \nu_{2}$ are analytic quotients of $S$ by the $H$ and $N$ actions respectively.

(ii) $\pi_{1}, \pi_{2}$ have dense range.

(iii) $\pi_{1}$ is surjective. $\pi_{2}$ is surjective $\Leftrightarrow S / H$ is analytic.

(iv) $\operatorname{Ker} \pi_{1}$ (resp. $\left.\operatorname{Ker} \pi_{2}\right)$ is q.e. to the $N$ (resp. H) action on $S$.

Proof. This is the content of the remainder of this section. 
Construction of the groupoid $\mathscr{G}$. The following general result is used at various points of our argument. The proof is related to that of [14] Theorem 6.17 .

Proposition 4.11. Let $Y, \mu$ be a measured analytic groupoid and let $X$ be an analytic Borel space which is also algebraically a groupoid. Let $T: Y \rightarrow X$ be a surjective Borel map such that

(i) $\quad(x, y) \in X^{(2)} \Rightarrow \exists(\xi, \eta) \in Y^{(2)}$ such that $T(\xi)=x, T(\eta)=y$.

(ii) $(\xi, \eta) \in Y^{(2)} \Rightarrow(T(\xi), T(\eta)) \in X^{(2)}$ and $T(\xi) T(\eta)=T(\xi \eta)$.

(iii) The fibre measures $\mu^{u}$ are strictly quasi-invariant and

$$
T(u)=T(v) \Rightarrow T_{*} \mu^{u} \sim T_{*} \mu^{v} \quad \forall u, v \in U_{Y}
$$

Then $X, T_{*}(\mu)$ is a measured analytic groupoid.

Proof. It is routine to check that all the groupoid operations on $X$ are Borel.

Clearly $\left[T_{*} \mu\right]$ is invariant under $i_{X}$, the inversion on $X$. $\bar{T}_{*} \bar{\mu}$.

Let $\bar{\mu}=\int_{U_{x}} \omega_{u} d \bar{T}_{*} \bar{\mu}$ be a decomposition of $\bar{\mu}$ with respect to $\bar{T}$, verifies

Define $\lambda^{u}=\int_{\bar{T}^{-1}(u)} T_{*} \mu^{\prime} d \omega_{u}(s), u \in U_{X}$. By direct integration one

$$
T_{*} \mu=\int_{U_{x}} \lambda^{u} d \bar{T}_{*} \bar{\mu}(u)
$$

By (iii), $\lambda^{u} \sim T_{*} \mu^{\prime} \forall s \in \bar{T}^{-1}(u)$. For $x \in X$, pick $\xi \in T^{-1}(x)$. Then $T_{*}^{x} \lambda^{d(x)} \sim T_{*}^{x} T_{*} \mu^{d(\xi)} \sim T_{*} \mu^{r(\xi)} \sim \lambda^{r(x)}$ where $T^{x}(y)=x y, y \in F^{d^{(x)}}$.

Thus $T_{*} \mu$ is a quasi-invariant measure on $X$.

Corollary 4.12. Suppose that in the situation of 4.11, (iii) is replaced by

(iii)' The $\mu^{\prime \prime}$ are strictly quasi-invariant and $\forall u, v \in U_{Y} u \sim v$, $T(u)=T(v) \Rightarrow \exists \xi \in Y$ with $T(\xi) \in U_{X}$ and $d(\xi)=u, r(\xi)=v$. Then (iii) is automatically satisfied.

Proof. Suppose $T(u)=T(v)$. Pick $\xi \in Y$ such that $T(\xi) \in U_{X}$, $d(\xi)=u, r(\xi)=v . \quad$ By 4.11.3, $T_{*} \mu^{v} \sim T_{*}^{T(\xi)} T_{*} \mu^{u}=T_{*} \mu^{u}$ since $T^{T(\xi)}=$ id.

We need some results about the stabilising subgroups of a group action. Let $G$ be a l.c.s.c. group and let $X$ be an analytic Borel $G$ space. The stabiliser in $G$ of a point $x \in X$ is known to be a closed 
subgroup $\sigma(x) \subseteq G,[1]$ p. 16. Let $\Sigma(G)$ denote the set of closed subgroups of $G$. $\Sigma(G)$ can be given a separable compact Hausdorff topology [6] and hence is also a standard Borel space. The action of $G$ on $\Sigma(G)$ by conjugation is jointly continuous, [1] p. 68. The map $\sigma: X \rightarrow \Sigma(G)$ is Borel and $G$ equivariant, [1] 1.58 .

Returning now to the situation of Theorem 4.10 , we simplify matters with the following lemmas:

Lemma 4.13. There are analytic quotients $S_{1}, S_{2}$ for the $H$ and $N$ actions on $S$ so that after removing an $N \times H$ invariant null set from $S, S$ is analytic and

(a) The spaces $S_{1}, S_{2}$ are analytic Borel $N \times H$ spaces, where the $H$ action on $S_{1}$ and the $N$ action on $S_{2}$ are trivial.

(b) The projection maps $p_{i}$ are Borel, $N \times H$ equivariant and surjective.

Proof. Find by 1.1 a standard quotient $S_{1}^{\prime}$ of $S$ by the $H$ action, which is an ergodic $N$ space. Let $H$ act trivially on $S_{1}^{\prime}$. The projection map $p_{1}^{\prime}$ is a.e. defined, Borel and almost $N \times H$ equivariant. By [14] Theorem 3.6 we may remove an $N \times H$ invariant null set $Y$ from $S$ so that $S-Y$ is analytic, and find a Borel $N \times H$ equivariant map $p_{1}$ : $S-Y \rightarrow S_{1}^{\prime}$ so that $p_{1}=p_{1}^{\prime}$ a.e.. Removing an $N \times H$ invariant null set from $S, p_{1}^{\prime}$ may be assumed surjective. $S / N$ is an analytic quotient $S_{2}$ of $S$ by the $N$ action. $S_{2}$ is clearly an analytic Borel $H$ space.

From now on we assume $S_{i}, p_{i}, S$ are as in Lemma 4.13 .

Lemma 4.14. Let $\sigma: S \rightarrow \Sigma(N)$ assign to each point of $S$ its stabilising subgroup in $N$. By removing an $N \times H$ invariant Borel null set from $S$ we may assume that $S$ is analytic and that there is a Borel $N$ equivariant map $\bar{\sigma}: S_{1} \rightarrow \Sigma(N)$ such that $\sigma=\bar{\sigma} p_{1}$.

Proof. $\sigma(s)=\sigma(s h) \forall s \in S, h \in H$. Therefore $\sigma$ induces a Borel map $\bar{\sigma}: S_{1} \rightarrow \Sigma(N)$ such that $\bar{\sigma} p_{1}(s)=\sigma(s)$ a.a. $s \in S$. Since $p_{1}$ and $\sigma$ are $N$ equivariant, $\bar{\sigma}$ is almost $N$ equivariant. Removing an $N$ invariant null set $E$ from $S_{1}$ and altering $\bar{\sigma}$ on a null set we may assume $S_{1}$ is analytic and $\bar{\sigma}$ is Borel and $N$ equivariant. $p_{1}^{-1}(E)$ is Borel, null, $N \times H$ equivariant, and has analytic complement in $S$, therefore may be removed. for $\bar{\sigma}$.

From now on we assume $\sigma, \bar{\sigma}$ are as in Lemma 4.14 and write $\sigma$

Set $\mathscr{A}=\left\{(t, \sigma(t) n): t \in S_{1}, n \in N\right\} . \mathscr{A}$ is given the structure of an algebraic groupoid by defining:

$$
d(t, \sigma(t) n)=t n, \quad r(t, \sigma(t) n)=t, \quad(t, \sigma(t) n)^{-1}=\left(t n, \sigma(t n) n^{-1}\right),
$$




$$
(t, \sigma(t) n)\left(t n, \sigma(t n) n^{\prime}\right)=\left(t, \sigma(t) n n^{\prime}\right) \quad \text { for } \quad t \in S_{1}, \quad n, n^{\prime} \in N .
$$

We write $s_{1}, s_{2}$ for $p_{1}(s), p_{2}(s)$ respectively if $s \in S$.

Lemma 4.15. There is a conull Borel Set $S_{1}^{*} \subseteq S_{1}$ so that $\mathscr{A}_{1}=\left.\mathscr{A}\right|_{S^{*}}$ is an analytic Borel space. (For the definition of $\mathscr{A}^{s_{i}^{*}} \mid s_{i}$ c.f. [14] p. 265, 290).

Proof. $P(S \times N)=\{(s, \sigma(s) n): s \in S, n \in N\}$ may be identified with the image of $S \times N$ in $S \times S$ under the Borel map $P:(s, n) \rightarrow(s, s n)$, and is therefore an analytic Borel space.

By von Neumann's selection lemma there are a conull Borel set $S_{1}^{*} \subseteq S_{1}$ and a Borel map $q: S_{1}^{*} \rightarrow S$ with

$$
p_{1} q(t)=t \quad t \in S_{1}^{*} .
$$

$B: \mathscr{A}_{1} \rightarrow S \times S, B(t, \sigma(t) n)=(q(t), q(t) n)$, is well defined and injective.

Set

$$
\begin{aligned}
& A=\left\{(s, s n) \in S \times S: s \in S, n \in N, s_{1}, s_{1} n \in S_{1}^{*}\right\} \\
& C=P\left(q\left(S_{1}^{*}\right) \times N\right)
\end{aligned}
$$

$A$ and $C$ are analytic and $B\left(\mathscr{A}_{1}\right)=C \cap A$.

Therefore $B^{-1}$ induces an analytic Borel structure on $\mathscr{A}_{1}$.

Proposition 4.16. $\quad \mathscr{A}_{1}$ is an analytic measured groupoid with measure $\nu_{1}$ on the units $S_{1}^{*}$.

Proof. We seek to apply 4.11. Set $\mathscr{A}_{1}^{\prime}=S \times\left. N\right|_{p_{1}^{-1}\left(S_{\mathbf{1}}^{*}\right)} T: \mathscr{A}_{1}^{\prime} \rightarrow \mathscr{A}_{1}$, $T(s, n)=\left(s_{1}, \sigma(s) n\right) . \quad T$ is surjective.

$B T(s, n)=\left(q\left(s_{1}\right), q\left(s_{1}\right) n\right)$. Therefore $B T$ is Borel, hence so is $T$.

We check the conditions of 4.11:

(i) $\quad\left((t, \sigma(t) n),\left(t^{\prime}, \sigma\left(t^{\prime}\right) n^{\prime}\right)\right) \in \mathscr{A}_{1}^{(2)} \Leftrightarrow t^{\prime}=t n .\left((q(t), n),\left(q(t) n, n^{\prime}\right)\right)$ $\in \mathscr{A}_{1}^{\prime(2)} \quad$ and $\quad T(q(t), n)=(t, \sigma(t) n) ; \quad T\left(q(t) n, n^{\prime}\right)=\left(t n, \sigma(t n) n^{\prime}\right)=$ $\left(t^{\prime}, \sigma\left(t^{\prime}\right) n^{\prime}\right)$.

(ii) $\quad\left((s, n),\left(s n, n^{\prime}\right)\right) \in \mathscr{A}_{1}^{\prime(2)} \quad \Rightarrow \quad\left(T(s, n), T\left(s n, n^{\prime}\right)\right) \in \mathscr{A}_{1}^{(2)} \quad$ and $T(s, n) T\left(s n, n^{\prime}\right)=T\left(s, n n^{\prime}\right)$.

(iii) Let $\lambda$ denote the natural measure on $\mathscr{A}_{1}^{\prime}$. The measures $\lambda^{u}$ are quasi-invariant $\forall u \in p_{1}^{-1}\left(S_{1}^{*}\right)$.

Suppose $u, v \in p_{1}^{-1}\left(S_{1}^{*}\right)$ with $\bar{T}(u)=\bar{T}(v)$.

$\lambda^{u}$ is in the restriction of the Haar class on $N$ to $\Lambda^{u}=$ $\left\{n \in N:(u, n) \in \mathscr{A}_{1}^{\prime}\right\} \bar{T}(u)=\bar{T}(v) \Rightarrow \Lambda^{u}=\Lambda^{v}$. Therefore $T_{*} \lambda^{u}=T_{*} \lambda^{v}$.

4.11 now shows that $\mathscr{A}_{1}, T_{*} \lambda$ is a measured analytic groupoid, and $\overline{T_{*} \lambda}=\bar{T}_{*} \nu=\nu_{1}$. 
$\mathscr{A}_{1}$ is algebraically a groupoid suitable for our purposes and we could at this point proceed to define the cocycles $\pi_{i}$. However these cocycles would not necessarily be normalised, a condition which is crucial for the fibre product construction of $S_{1} * S_{2} * \mathscr{G}$. This difficulty is circumvented by restricting $\mathscr{A}$ to a suitable subset of $S_{1}$ (c.f. [14] p. 265 and p. 290) which will ensure that $\pi_{1}$ and $\pi_{2}$ are normalised.

The appropriate set is a Borel set which intersects each $N$ orbit on $S_{1}$ at most couritably often. More precisely: Let $G$ be a l.c.s.c. group and let $S$ be an analytic Borel $G$ space with quasi-invariant measure v. $E \in \mathscr{B}(S)$ is a canonical section for the action of $G$ on $S$ if there is a conull Borel set $S^{*} \subseteq S$, and a Borel map $T: S^{*} \rightarrow E$ with $T(s)=s g$ for some $g \in G, \forall s \in S^{*}$, and so that $E$ intersects each $G$ orbit at most countably many times.

Remark (1). Set $\omega=T_{*}(\nu)$. Since the relation induced on $E$ is countable, by [4] Theorem 1 , for $A \in \mathscr{B}(E)$ :

$$
\omega(A)=0 \Leftrightarrow \omega([A])=0 \Leftrightarrow \nu\left(T^{-1}([A])\right)=0 \Leftrightarrow A \text { is negligible (2.5), }
$$

i.e., the null sets of $\omega$ are precisely the $\nu$ negligible sets of $E$.

REMARK (2). It is not hard to prove that if there is a canonical section for the $G$ action on $S$, and if $T \subseteq S$ is conull, then there is a canonical section contained in $T$.

The existence of canonical sections for actions of l.c.s.c. groups was proved by the author in [17] whenever the map $\sigma: S \rightarrow \Sigma(G)$ is continuous. The result has since been extended in [5] to the general case.

Choose a canonical section $Z \subseteq S_{1}^{*}$, a conull Borel subset $S_{1}^{* *} \subseteq S_{1}$, and a Borel map $\alpha: S_{1}^{* *} \rightarrow N$, so that $\bar{\alpha}(s)=s \alpha(s) \in Z \forall s \in S_{1}^{* *}$. Set $\mathscr{A}_{2}=\left.\mathscr{A}\right|_{s_{i}^{*}}, \mathscr{G}=\left.\mathscr{A}\right|_{z}$

PropostTION 4.17. $G$ is an analytic measured groupoid with measure $\bar{\alpha}_{*}\left(\nu_{1}\right)$ on the units $Z$.

Proof. This follows from Theorem 6.17 of [14].

We modify the measure on $\mathscr{G}$ to ensure that it is symmetric, and use the standard notation of 2 .

The cocycles $\pi_{1}$ and $\pi_{2}$.

The following Lemma is useful: 
Lemma 4.18. Let $X, Y$ be standard Borel spaces and let $Z$ be an analytic Borel space. Let $F: X \times Y \rightarrow Z$ be a Borel map. Suppose $A \in \mathscr{B}(X \times Y)$, and that the projection of $A$ on $X$ is $X$. Suppose that

$$
(x, y),\left(x, y^{\prime}\right) \in A \Rightarrow F(x, y)=F\left(x, y^{\prime}\right) \text {. }
$$

Then $f(x)=F(x, y),(x, y) \in A$, is a well defined Borel function $X \rightarrow Z$.

Proof. It is clear that $f$ is well defined. Suppose $E \in \mathscr{B}(Z)$. $f^{-1}(E)=P\left(F^{-1}(E) \cap A\right)$, where $P: X \times Y \rightarrow X$ is projection. Therefore $f^{-1}(E)$ is analytic. Also $X-f^{-1}(E)=f^{-1}(Z-E)$ and so is analytic. By the separation theorem [1] p. $6, f^{-1}(E)$ is Borel.

$p_{2}\left(p_{1}^{-1}\left(S_{1}^{* *}\right)\right)$ is analytic and conull in $S_{2}$, so by von Neumann's selection lemma there is a conull Borel set $S_{2}^{*} \subseteq S_{2}$ and a Borel map $\delta: S_{2} \rightarrow S$ so that

$$
p_{2} \delta(t)=t \quad \text { and } \quad \delta(t) \in p_{1}^{-1}\left(S_{1}^{* *}\right) \quad \forall t \in S_{2}^{*}
$$

Define $\epsilon: S_{2}^{*} \rightarrow N, \epsilon(t)=\alpha p_{1} \delta(t)$ and $\bar{\epsilon}: S_{2}^{*} \rightarrow Z, \bar{\epsilon}(t)=\bar{\alpha} p_{1} \delta(t)$.

LEMMA 4.19. $\quad \bar{\epsilon}_{*}\left(\nu_{2}\right)$ is absolutely continuous with respect to $\bar{\alpha}_{*}\left(\nu_{1}\right)$.

Proof. For $E \in \mathscr{B}(Z)$ :

$$
\begin{aligned}
\bar{\alpha}_{*} \nu_{1}(E) & =0 \Rightarrow \nu_{1}([E])=0 \Rightarrow \nu\left(p_{1}^{-1}([E])\right)=0 \Rightarrow \nu\left(\left[p_{1}^{-1}(E)\right]\right)=0 \\
& \Rightarrow \delta_{*} \nu_{2}\left(\left[p_{1}^{-1}(E)\right]\right)=0 \\
& \Rightarrow p_{1 *} \delta_{*} \nu_{2}([E])=0 \Rightarrow \bar{\alpha}_{*} p_{1 *} \delta_{*} \nu_{2}(E)=0 \Rightarrow \bar{\epsilon}_{*} \nu_{2}(E)=0 .
\end{aligned}
$$

Now modify $\alpha, \epsilon$ to maps $\alpha^{\prime}, \epsilon^{\prime}$ so that $\bar{\alpha}_{*}^{\prime}\left(\nu_{1}\right) \sim \bar{\epsilon}_{*}^{\prime}\left(\nu_{2}\right)$ as follows: find $\gamma: Z \rightarrow N$ such that $\gamma(z)=e, z \in \operatorname{supp} \bar{\epsilon}_{*}\left(\nu_{2}\right) ; z \gamma(z) \in \operatorname{supp} \bar{\epsilon}_{*}\left(\nu_{2}\right)$. Set $\alpha^{\prime}=\gamma \circ \alpha, \epsilon^{\prime}=\gamma \circ \epsilon$. Write $\alpha, \epsilon$ for $\alpha^{\prime}, \epsilon^{\prime}$.

4.20. Construction of $\pi_{1}$ and $\pi_{2}$. Set $L=S_{1} \times\left. N\right|_{s_{*}^{*} .} L$ is an i.c. of $S_{1} \times N$. Define $\pi_{1}: L \rightarrow \mathscr{G}, \pi_{1}(u, n)=\left(\bar{\alpha}(u), \sigma(\bar{\alpha}(u)) \alpha(u)^{-1} n \alpha(u n)\right) . \pi_{1}$ can easily be checked to be Borel (c.f. 4.17). Moreover $\bar{\alpha}(u) \in Z$ and $\bar{\alpha}(u) \alpha(u)^{-1} n \alpha(u n)=\bar{\alpha}(u n) \in Z$, so that $\pi_{i}(u, n) \in \mathscr{G}$.

Set $K=S_{2} \times\left. H\right|_{s \xi}$. $K$ is an i.c. of $S_{2} \times H . \quad$ For $(t, h) \in K$ :

$$
p_{2} \delta(t h)=t h=p_{2} \delta(t) h=p_{2}(\delta(t) h) .
$$

Since $S_{2}=S / N, \quad \delta(t h) \in \delta(t) h N$. Let $A=\{(t, h, n) \in K \times N: \delta(t h)=$ $\delta(t) h n\}$. Write $\beta(t, h)$ for any element of $N$ such that $(t, h, \beta(t, h)) \in A$. $A$ is Borel. Set $F: K \times N \rightarrow \mathscr{G}, F(t, h, n)=\left(\bar{\epsilon}(t), \sigma \tilde{\epsilon}(t) \epsilon(t)^{-1} n \epsilon(t h)\right)$. It is 
easy to check that $F(t, h, n) \in \mathscr{G}$. Moreover one verifies that $(t, h, n)$, $\left(t, h, n^{\prime}\right) \in A \Rightarrow F(t, h, n)=F\left(t, h, n^{\prime}\right)$. Therefore by Lemma 4.18 and the definition of the Borel structure on $\mathscr{G}, \pi_{2}(t, h)=$ $\left(\tilde{\epsilon}(t), \sigma(\bar{\epsilon}(t)) \epsilon(t)^{-1} \beta(t, h) \epsilon(t h)\right)$ is Borel.

LEMMA 4.21. $\pi_{1}: L \rightarrow \mathscr{G}$ and $\pi_{2}: K \rightarrow \mathscr{G}$ are strict normalized Borel cocycles.

Proof. (a)

$$
\begin{aligned}
& (u, n) \in L,\left(u n, n^{\prime}\right) \in L \Rightarrow \pi_{1}(u, n) \pi_{1}\left(u n, n^{\prime}\right) \\
& \quad=\left(\bar{\alpha}(u), \sigma(\bar{\alpha}(u)) \alpha(u)^{-1} n \alpha(u n)\right)\left(\bar{\alpha}(u n), \sigma(\bar{\alpha}(u n)) \alpha(u n)^{-1} n^{\prime} \alpha\left(u n n^{\prime}\right)\right) \\
& \quad=\left(\bar{\alpha}(u), \sigma(\bar{\alpha}(u)) \alpha(u)^{-1} n n^{\prime} \alpha\left(u n n^{\prime}\right)\right)=\pi_{1}\left(u, n n^{\prime}\right)
\end{aligned}
$$

$$
\begin{aligned}
& (t, h) \in K,\left(t h, h^{\prime}\right) \in K \Rightarrow \pi_{2}(t, h) \pi_{2}\left(t h, h^{\prime}\right) \\
& =\left(\bar{\epsilon}(t), \sigma(\bar{\epsilon}(t)) \epsilon(t)^{-1} \beta(t, h) \epsilon(t h)\right) \\
& \quad \times\left(\bar{\epsilon}(t h), \sigma(\bar{\epsilon}(t h)) \epsilon(t h)^{-1} \beta\left(t h, h^{\prime}\right) \epsilon\left(t h h^{\prime}\right)\right) \\
& =\left(\bar{\epsilon}(t), \sigma(\bar{\epsilon}(t)) \epsilon(t)^{-1} \beta(t, h) \beta\left(t h, h^{\prime}\right) \epsilon\left(t h h^{\prime}\right)\right) .
\end{aligned}
$$

Now

$$
\begin{aligned}
\delta\left(t h h^{\prime}\right) & =\delta(t h) h^{\prime} \beta\left(t h, h^{\prime}\right)=\delta(t) h \beta(t, h) h^{\prime} \beta\left(t h, h^{\prime}\right) \\
& =\delta(t) h h^{\prime} \beta(t, h) \beta\left(t h, h^{\prime}\right) .
\end{aligned}
$$

Therefore we may choose $\beta\left(t, h h^{\prime}\right)=\beta(t, h) \beta\left(t h, h^{\prime}\right)$, so that

$$
\begin{aligned}
\pi_{2}\left(t, h h^{\prime}\right) & =\left(\bar{\epsilon}(t), \sigma(\bar{\epsilon}(t)) \epsilon(t)^{-1} \beta(t, h) \beta\left(t h, h^{\prime}\right) \epsilon\left(t h h^{\prime}\right)\right) \\
& =\pi_{2}(t, h) \pi_{2}\left(t h, h^{\prime}\right) .
\end{aligned}
$$

(c) $\bar{\pi}_{1 *} \nu_{1}=\bar{\alpha}_{*} \nu_{1} \sim \bar{\mu}$ and $\bar{\pi}_{2 *} \nu_{2}=\bar{\epsilon}_{*} \nu_{2} \sim \bar{\alpha}_{*} \nu_{1}$ by Lemma 4.19.

Now define $\Sigma=S_{1} * S_{2} * \mathscr{G}$ as in 4.1. $\Sigma$ is an almost $N \times H$ space by Lemma 4.4.

Isomorphism of $S$ with $\Sigma$. We show that the $N \times H$ space $S$ is almost isomorphic to the almost $N \times H$ space $\Sigma$.

4.22. Construction of the almost isomorphism $P$. Let $J=S \times\left. N\right|_{p \bar{z}^{-1}\left(S^{*}\right)}$ and $A=\left\{(s, n) \in J:\left(\delta p_{2}(s)\right) n=s\right\}$. Now 


$$
\begin{array}{ll}
p_{2} \delta p_{2}(s)=p_{2}(s) & \forall s \in p_{2}^{-1}\left(S_{2}^{*}\right), \\
\delta p_{2}(s) \in s N & \forall s \in p_{2}^{-1}\left(S_{2}^{*}\right) .
\end{array}
$$

Therefore the projection of $A$ on $p_{2}^{-1}\left(S_{2}^{*}\right)$ is $p_{2}^{-1}\left(S_{2}^{*}\right) . \quad A$ is Borel. Set $F: J \rightarrow S, F(s, n)=q \tilde{\epsilon}\left(s_{2}\right) \epsilon\left(s_{2}\right)^{-1} n . \quad F$ is Borel and $(s, n),\left(s, n^{\prime}\right) \in A \Rightarrow$ $s=\delta p_{2}(s) n=\delta p_{2}(s) n^{\prime} \Rightarrow n n^{\prime-1} \in \sigma(s)=\sigma\left(q\left(\bar{\epsilon}\left(s_{2}\right)\right) \epsilon\left(s_{2}\right)^{-1}\right) \Rightarrow F(s, n)=$ $F\left(s, n^{\prime}\right)$.

Write $k(s)$ for any element of $N$ such that $(s, k(s)) \in A$. By Lemma 4.18, $f: p_{2}^{-1}\left(S_{2}^{*}\right) \rightarrow S, f(s)=q\left(\bar{\epsilon}\left(s_{2}\right)\right) \epsilon\left(s_{2}\right)^{-1} k(s)$, is Borel.

Set $T=p_{2}^{-1}\left(S_{2}^{*}\right) \cap p_{1}^{-1}\left(S_{1}^{* *}\right) . \quad T$ is conull in $S$. Let $R=$ $S_{1}^{* *} * S_{2}^{*} * \mathscr{G} \subseteq \Sigma . \quad R$ is conull in $\Sigma$. Define $P: T \rightarrow R$,

$$
P(s)=\left(s_{1}, s_{2} ; \bar{\epsilon}\left(s_{2}\right), \sigma\left(\bar{\epsilon}\left(s_{2}\right)\right) \epsilon\left(s_{2}\right)^{-1} k(s) \alpha\left(s_{1}\right)\right) .
$$

We check that $P(s) \in R$ :

$$
\begin{gathered}
s \in T \Rightarrow s_{1} \in S_{1}^{* *}, \quad s_{2} \in S_{2}^{*} . \\
\bar{\pi}_{2}\left(s_{2}\right)=\bar{\epsilon}\left(s_{2}\right), \quad \bar{\pi}_{1}\left(s_{1}\right)=\bar{\alpha}\left(s_{1}\right), \\
r\left(\bar{\epsilon}\left(s_{2}\right), \sigma\left(\bar{\epsilon}\left(s_{2}\right)\right) \epsilon\left(s_{2}\right)^{-1} k(s) \alpha\left(s_{1}\right)\right)=\bar{\epsilon}\left(s_{2}\right), \\
d\left(\bar{\epsilon}\left(s_{2}\right), \sigma\left(\bar{\epsilon}\left(s_{2}\right)\right) \epsilon\left(s_{2}\right)^{-1} k(s) \alpha\left(s_{1}\right)\right)=\bar{\epsilon}\left(s_{2}\right) \epsilon\left(s_{2}\right)^{-1} k(s) \alpha\left(s_{1}\right) \\
=p_{1} \delta\left(s_{2}\right) k(s) \alpha\left(s_{1}\right)=p_{1}\left(\delta\left(s_{2}\right) k(s)\right) \alpha\left(s_{1}\right)=p_{1}(s) \alpha\left(s_{1}\right)=\bar{\alpha}\left(s_{1}\right) .
\end{gathered}
$$

$P$ is Borel $\Leftrightarrow p_{1} \times p_{2} \times(B \cdot P): S \rightarrow S_{1} \times S_{2} \times S \times S$ is Borel $\Leftrightarrow$ $s \mapsto\left(q \bar{\epsilon}\left(s_{2}\right), q \bar{\epsilon}\left(s_{2}\right) \epsilon\left(s_{2}\right)^{-1} k(s) \alpha\left(s_{1}\right)\right)$ is Borel $\Leftrightarrow s \mapsto\left(q \bar{\epsilon}\left(s_{2}\right), f(s) \alpha\left(s_{1}\right)\right)$ is Borel.

$q, \bar{\epsilon}, f, \alpha$ are already known to be Borel, hence so is $P$.

LEMMA 4.23. $P: T \rightarrow R$ is bijective.

Proof. For $(x, y ; \bar{\epsilon}(y), \sigma(\bar{\epsilon}(y)) m) \in \Sigma$ note that

$$
\bar{\alpha}(x)=\bar{\epsilon}(y) m, \quad \text { i.e., } \quad x=\bar{\epsilon}(y) m \alpha(x)^{-1} .
$$

Define $Q: R \rightarrow T$ by

$$
Q(x, y ; \bar{\epsilon}(y), \sigma(\bar{\epsilon}(y)) m)=\delta(y) \epsilon(y) m \alpha(x)^{-1} .
$$

Since $\sigma(\bar{\varepsilon}(y))=\sigma\left(p_{2} \delta(y) \epsilon(y)\right)=\sigma(\delta(y) \epsilon(y))$ this is well defined. For $s \in T:$

$$
\begin{aligned}
Q P(s) & =Q\left(s_{1}, s_{2} ; \bar{\epsilon}\left(s_{2}\right), \sigma\left(\bar{\epsilon}\left(s_{2}\right)\right) \epsilon\left(s_{2}\right)^{-1} k(s) \alpha\left(s_{1}\right)\right) \\
& =\delta\left(s_{2}\right) \epsilon\left(s_{2}\right) \epsilon\left(s_{2}\right)^{-1} k(s) \alpha\left(s_{1}\right) \alpha\left(s_{1}\right)^{-1}=\delta\left(s_{2}\right) k(s)=s .
\end{aligned}
$$


For $(x, y ; \bar{\epsilon}(y), \sigma(\bar{\epsilon}(y)) m) \in R$ :

$$
\begin{aligned}
P Q(x, y ; \bar{\epsilon}(y), \sigma(\bar{\epsilon}(y)) m) & =P\left(\delta(y) \epsilon(y) m \alpha(x)^{-1}\right) . \\
p_{1}\left(\delta(y) \epsilon(y) m \alpha(x)^{-1}\right) & =p_{1} \delta(y) \epsilon(y) m \alpha(x)^{-1} \\
& =\bar{\epsilon}(y) m \alpha(x)^{-1} \\
& =x
\end{aligned}
$$

$p_{2}\left(\delta(y) \epsilon(y) m \alpha(x)^{-1}\right)=p_{2} \delta(y)=y$.

Also since $\delta p_{2}\left(\delta(y) \epsilon(y) m \alpha(x)^{-1}\right)=\delta(y)$ we may take $k\left(\delta(y) \epsilon(y) m \alpha(x)^{-1}\right)=\epsilon(y) m \alpha(x)^{-1}$. Therefore

$$
\begin{aligned}
P\left(\delta(y) \epsilon(y) m \alpha(x)^{-1}\right) & =\left(x, y ; \bar{\epsilon}(y), \sigma(\bar{\epsilon}(y)) \epsilon(y)^{-1} \epsilon(y) m \alpha(x)^{-1} \alpha(x)\right) \\
& =(x, y ; \bar{\epsilon}(y), \sigma(\bar{\epsilon}(y)) m) .
\end{aligned}
$$

LEMma 4.24. P is almost $N$ equivariant and almost $H$ equivariant.

Proof. (a) $s \in T \Rightarrow \delta\left(s_{2}\right) \in T$ and

$$
\begin{aligned}
& P\left(\delta\left(s_{2}\right)\right) k(s)=\left(p_{1} \delta\left(s_{2}\right), s_{2} ; \bar{\epsilon}\left(s_{2}\right), \sigma\left(\bar{\epsilon}\left(s_{2}\right)\right) \epsilon\left(s_{2}\right)^{-1} \alpha p_{1} \delta\left(s_{2}\right)\right) k(s) \\
& =\left(p_{1} \delta\left(s_{2}\right) k(s), s_{2} ;\right. \\
& \left.\quad \bar{\epsilon}\left(s_{2}\right), \sigma\left(\bar{\epsilon}\left(s_{2}\right)\right) \epsilon\left(s_{2}\right)^{-1} \alpha\left(p_{1} \delta\left(s_{2}\right)\right) \alpha p_{1} \delta\left(s_{2}\right)^{-1} k(s) \alpha\left(p_{1} \delta\left(s_{2}\right) k(s)\right)\right) \\
& =\left(s_{1}, s_{2} ; \bar{\epsilon}\left(s_{2}\right), \sigma\left(\bar{\epsilon}\left(s_{2}\right)\right) \epsilon\left(s_{2}\right)^{-1} k(s) \alpha\left(s_{1}\right)\right) \\
& =P(s) .
\end{aligned}
$$

Therefore $\quad s \in T, \quad m \in N, \quad s m \in T \Rightarrow P(s m)=P\left(\delta p_{2}(s m)\right) k(s m)=$ $P\left(\delta\left(s_{2}\right)\right) k(s) m=P(s) m$.

(b) $s \in T, h \in H$, sh $\in T$

$$
\begin{aligned}
\Rightarrow & \bar{\epsilon}\left(s_{2}\right) \epsilon\left(s_{2}\right)^{-1} \beta\left(s_{2}, h\right) \epsilon\left(s_{2} h\right)=p_{1} \delta\left(s_{2}\right) \beta\left(s_{2}, h\right) \epsilon\left(s_{2} h\right) \\
& =p_{1} \delta\left(s_{2} h\right) \epsilon\left(s_{2} h\right)=\bar{\epsilon}\left(s_{2} h\right) \\
\Rightarrow & P(s) h=\left(s_{1}, s_{2} ; \bar{\epsilon}\left(s_{2}\right), \sigma\left(\bar{\epsilon}\left(s_{2}\right)\right) \epsilon\left(s_{2}\right)^{-1} k(s) \alpha\left(s_{1}\right)\right) h \\
& =\left(s_{1}, s_{2} h ;\right. \\
\left(\bar{\epsilon}\left(s_{2}\right), \sigma\left(\bar{\epsilon}\left(s_{2}\right)\right)\right. & \left.\left.\epsilon\left(s_{2}\right)^{-1} \beta\left(s_{2}, h\right) \epsilon\left(s_{2} h\right)\right)^{-1}\left(\bar{\epsilon}\left(s_{2}\right), \sigma\left(\bar{\epsilon}\left(s_{2}\right)\right) \epsilon\left(s_{2}\right)^{-1} k(s) \alpha\left(s_{1}\right)\right)\right) \\
& =\left(s_{1}, s_{2} h ; \bar{\epsilon}\left(s_{2} h\right), \sigma\left(\bar{\epsilon}\left(s_{2} h\right)\right) \epsilon\left(s_{2} h\right)^{-1} \beta\left(s_{2}, h\right)^{-1} k(s) \alpha\left(s_{1}\right)\right) \\
& =\left(s_{1}, s_{2} h ; \bar{\epsilon}\left(s_{2} h\right), \sigma\left(\bar{\epsilon}\left(s_{2} h\right)\right) \epsilon\left(s_{2} h\right)^{-1} k(s h) \alpha\left(s_{1}\right)\right)=P(s h)
\end{aligned}
$$


the penultimate equality following because

$$
s h=\delta\left(s_{2}\right) k(s) h=\delta\left(s_{2}\right) h k(s)=\delta\left(s_{2} h\right) \beta\left(s_{2}, h\right)^{-1} k(s)
$$

so that we may take $k(s h)=\beta\left(s_{2}, h\right)^{-1} k(s)$.

To complete the proof that $P$ is an almost isomorphism we need

Proposition 4.25. $P_{*} \nu \sim m$.

Proof. We have $q_{2} P=p_{2}$ where $q_{2}: \Sigma \rightarrow S_{2}$ is projection and $P: T \rightarrow R \quad$ satisfies $P(s n)=P(s) n ; s, s n \in T$ and $P(s h)=P(s) h$; $s, s h \in R$. Therefore $\nu_{2}=p_{2 *} \nu=q_{2 *} P_{*} \nu$. By [14], p. 266, $q_{2 *} m=\nu_{2}$, so it is sufficient to show that if $m=\int_{S_{2}} m_{t} d \nu_{2}(t)$ and $\nu=\int_{S_{2}} \nu_{t} d \nu_{2}(t)$ are decompositions of $m$ with respect to $p_{2}, q_{2}$ respectively then $m_{t} \sim P_{*} \nu_{t}$ a.a. $t \in S_{2}$.

The level sets of $\left.q_{2}\right|_{R}$ are the intersections of $N$ orbits with $R$; hence the level sets of $\left.q_{2}\right|_{R N}$ are orbits and $q_{2}$ is an ergodic decomposition. The measures $\nu_{t}, m_{t}$ are quasi-invariant by [1] p. 72. Hence $P_{*} m_{t}$ is quasi-invariant and therefore equivalent to $\nu_{t}$ a.a. $t$.

We are finally in a position to prove the main result:

Proof of Theorem 4.10. Construct $S_{1}, S_{2}, \pi_{1}, \pi_{2}, \mathscr{G}, \Sigma$ as in 4.11-4.21. The isomorphism of the $N \times H$ action on $S$, $\nu$ with $\tilde{M}\left(S_{1}, \pi_{1}, S_{2}, \pi_{2}, \mathscr{G}\right)$ follows from $4.23,4.25,4.24$ and 1.1 .

(i) This is clear by construction.

(ii) We show $\pi_{1}$ has dense range.

As in $4.25, q_{2}$ is an ergodic decomposition of the $N$ action on $\Sigma$. Let $R: X^{*}\left(\pi_{1}\right) \rightarrow Z, R(u, \xi)=r(\xi)$. By [14] p. 266, $R_{*}\left(\omega\left(\pi_{1}\right)\right)=r_{*} \mu=$ $\bar{\mu}$. Let $\omega\left(\pi_{1}\right)=\int_{Z} \omega_{v} d \bar{\mu}(v)$ be a decomposition of $\omega\left(\pi_{1}\right)$ with respect to $R, \bar{\mu}$.

$q_{2}^{-1}(t)=\{t\} \times\left(S_{1} *_{\bar{\alpha}, d} F_{\bar{\epsilon}(t)}\right)$ where $F_{\bar{\epsilon}(t)}=d^{-1}(\bar{\epsilon}(t))$, and by [14] p. $266 m_{t}$ is the measure $\omega_{\bar{\epsilon}(t)}$ a.a. $t \in Z$. Moreover the almost $N$ actions on $q_{2}^{-1}(t)$, $m_{t}$ and $R^{-1}(\bar{\epsilon}(t)), \omega_{\bar{\epsilon}(t)}$ may clearly be identified. Hence $R$ is an ergodic decomposition of $X^{*}\left(\pi_{1}\right)$.

(iii) As in 4.25 , the almost $N$ action on $q_{2}^{-1}(t), m_{t}$ is essentially transitive so that $\pi_{1}$ is surjective.

$\pi_{2}$ is surjective $\Leftrightarrow X\left(\pi_{2}\right) / H$ is analytic

$$
\begin{aligned}
& \Leftrightarrow S_{1} *\left(X\left(\pi_{2}\right) / H\right) \text { is analytic } \\
& \Leftrightarrow \Sigma / H \text { is analytic } \Leftrightarrow S / H \text { is analytic. }
\end{aligned}
$$

(iv) This is Lemma 4.8 . 


\section{Concluding remarks.}

REMARK 5.1. If $N$ is compact any action of $N$ is smooth. In particular, if $S$ is an ergodic $N \times H$ space, $S / N$ is analytic (compare Proposition 3.4). Moreover in this case $\mathscr{G}$ may be taken to be a l.c.s.c. group $G$ and the fibre product $S_{1} * S_{2} * \mathscr{G}$ reduces to a direct product $S_{1} \times S_{2} \times G . \quad S_{1}$ is a transitive $G$ space. Let one of the stabilising subgroups be $J$. All stabilising subgroups of the $N$ action on $S$ are conjugate. (Consider $\sigma: S \rightarrow \Sigma(N)$ which is constant on $H$ orbits and use the fact that $\Sigma(N)$ is smooth, [1] Appendix A.) Let $L$ be one such stabiliser with $L \subseteq J$. Then $L$ is normal in $J$ and we may take $G=J / L$. The proof of 4.10 is simplified considerably in this case.

ExAmple 5.2. Let $N, H$ be 1.c.s.c. groups, $S_{1}$ an ergodic $N$ space, $S_{2}=\{p t\},. \quad G=H, \quad \pi: S_{1} \times N \rightarrow G$ a Borel cocycle with dense range. Then the $N \times H$ action $M\left(S_{1}, \pi_{1}, p t, i d, H\right)$ restricted to $N$ is Ker $\pi$, often called the skew product of $S_{1}$ with $G$ by $\pi$. If $H$ is compact and abelian, these actions are the compact abelian group extensions of Parry.

Remark 5.3. For an $N \times H$ action to be representable, the $N$ action need not necessarily be smooth. (c.f. Remark 3.13). E.g.:

EXAmPle 5.4. $N=H=\mathbf{R} ; G=\mathbf{T}^{2}$, the two dimensional torus; $S_{1}=S_{2}=p t ; \pi_{1}: \mathbf{R} \rightarrow \mathbf{T}^{2}, \pi_{1}(t)=\left(e^{i t}, e^{2 \pi i t}\right) ; \pi_{2}: \mathbf{R} \rightarrow \mathbf{T}^{2}, \pi_{2}(t)=\left(e^{i t}, e^{2 \pi i \alpha t}\right)$ where $1,2 \pi, 2 \pi \alpha$ are independent over $Q$.

The $\mathbf{R} \times \mathbf{R}$ action on $\mathbf{T}^{2}, M\left(\pi_{1}, \pi_{2}, T^{2}\right)$, is a model but neither $\Sigma / N$ nor $\Sigma / H$ is analytic.

More generally whenever $S_{1}=S_{2}=\{p t$. $\}$ representable actions are those of the form $M\left(\pi_{1}, \pi_{2}, G\right)$ where $\pi_{1}: N \rightarrow G$ and $\pi_{2}: H \rightarrow G$ are homomorphisms with dense range. Such actions are closely related to actions with pure point spectrum.

REMARK 5.5. $\mathscr{G}$ cannot always be replaced by a l.c.s.c. group $G$. At the opposite extreme we have the following example, in which $\mathscr{G}$ is principal:

EXAMPle 5.6. Let $\mathbf{Z}$ act on $\mathbf{T}$ by rotation through an irrational angle $\alpha$. Let $\mathscr{G}=\{(\theta, \theta+n \alpha) \in \mathbf{T} \times \mathbf{T}: \theta \in \mathbf{T}, n \in \mathbf{Z}\} ; S_{1}=S_{2}=T ; N=$ $H=\mathbf{Z}$; let the actions of $N, H$ on $\mathbf{T}$ be rotations by $\alpha$; and let $\pi_{1}(\theta, n)=\pi_{2}(\theta, n)=(\theta, \theta+n \alpha)$.

Then $\Sigma=\{(\theta, \theta+n \alpha) \in \mathbf{T} \times \mathbf{T}: \theta \in T, n \in Z\}(\theta, \theta+n \alpha)\left(n_{1}, n_{2}\right)=$ $\left(\theta+n_{1} \alpha_{1}, \theta+n \alpha+n_{2} \alpha_{2}\right)$. It is clear that there is no group such that $\Sigma=T \times T \times G$. Notice that in this case both $\Sigma / N$ and $\Sigma / H$ are analytic. 
Example 5.6 can be regarded as a fibering of $T$ with itself over an equivalence relation on itself. More generally whenever $\mathscr{G}$ is principal, $M$ is a fibering of $S_{1}$ and $S_{2}$ over an equivalence relation on $U_{s \varphi}$. The general model is a combination of this situation with that of Example 5.4. Above each point of the space $S_{1} * S_{2}$ is a subset $\Gamma_{u, v}$ of $\mathscr{G}$ (c.f. 2.4). In particular if $(x, y) \in S_{1} * S_{2}$ and $\bar{\pi}_{1}(x)=\bar{\pi}_{2}(y)=u$ then if we set $Q(x, y)=\left\{s \in \Sigma: s_{1}=x, s_{2}=y\right\}, \quad \sigma_{N}(x) \times \sigma H(y) \subseteq N \times H$ fixes $Q(x, y)$ and the action is of the type described in 5.4.

REMARK 5.7. $\mathscr{G}$ is in general not unique. This is seen for example by the arbitrariness in the choice of $Z$ on p. 41 . $\mathscr{G}$ is however unique up to similarity.

It would be of interest to give a complete description of closed subgroups along the lines of 3 and to see to what extent this generalised to the ergodic case.

\section{REFERENCES}

1. L. Auslander and C. C. Moore, Unitary representations of solvable Lie groups, Mem. Amer. Math. Soc., 62 (1966).

2. S. Banach, Théorie des opérations linéaires, Monogram. Mat., Tom 1, Warsaw (1932).

3. J. Dixmier, Dual et quasi dual d'une algèbre de Banach involutive, Trans. Amer. Math. Soc., 107 (1963), 83-106.

4. J. Feldman and C. C. Moore, Ergodic equivalence relations, cohomology and von Neumann algebras, Preprint.

5. J. Feldman, P. Hahn and C. C. Moore, Measure groupoids coming from group actions, Notices Amer. Math. Soc., April 1976, 735-B17.

6. J. M. G. Fell, A Hausdorff topology for the closed subsets of a locally compact non-Hausdorff space, Proc. Amer. Math. Soc., 13 (1962), 472-476.

7. P. Forrest, Virtual subgroups of $R^{n}$ and $Z^{n}$, Adv. Math., 3 (1974), 187-207.

8. P. Hahn, The regular representations of measure groupoids, Preprint.

9. J. Kelley, General Topology, Van Nostrand, New York, 1955.

10. G. W. Mackey, Ergodic theory and virtual groups, Math. Annalen, 166 (1966), 187-207.

11. Borel structures in groups and their duals, Trans. Amer. Math. Soc., 85 (1957), 134-165.

12. - Point realisations of transformation groups, Illinois J. Math., 6 (1962), 327-335.

13. Induced representations of locally compact groups I, Ann. Math., 55 (1952), 101-139.

14. A. Ramsay, Virtual groups, Adv. Math., 6 (1971), 253-321.

15. Boolean duals of virtual groups, J. Functional Anal., 15 (1974), 56-101.

16. - Subobjects of virtual groups, Preprint.

17. C. Series, Ergodic actions of product groups, Thesis, Harvard, May 1976.

18. V. S. Varadarajan, Geometry of Quantum Theory, Vol. II, Van Nostrand, New York, 1970.

19. J. Westman, Virtual group homomorphisms with dense range, Illinois J. Math., 20 (1976), 41-47.

Received March 15, 1977.

UNIVERSITY OF CALIFORNIA

Berkeley, CA 94720 



\section{PACIFIC JOURNAL OF MATHEMATICS \\ EDITORS}

RICHARD ARENS (Managing Editor)

University of California

Los Angeles, CA 90024

\section{R. A. Beaumont \\ University of Washington \\ Seattle, WA 98105}

C. C. MOORE

University of California

Berkeley, CA 94720

\section{J. DUGUNDJI}

Department of Mathematics

University of Southern California

Los Angeles, CA 90007

R. FINN AND J. MILGRAM

Stanford University

Stanford, CA 94305

\section{ASSOCIATE EDITORS}

\section{E. F. BECKENBACH}

B. H. NeUmanN

F. WOLF

K. YoSHIDA

\section{SUPPORTING INSTITUTIONS}

\author{
UNIVERSITY OF BRITISH COLUMBIA \\ CALIFORNIA INSTITUTE OF TECHNOLOGY \\ UNIVERSITY OF CALIFORNIA \\ MONTANA STATE UNIVERSITY \\ UNIVERSITY OF NEVADA \\ NEW MEXICO STATE UNIVERSITY \\ OREGON STATE UNIVERSITY \\ UNIVERSITY OF OREGON \\ OSAKA UNIVERSITY
}

\author{
UNIVERSITY OF SOUTHERN CALIFORNIA \\ STANFORD UNIVERSITY \\ UNIVERSITY OF HAWAII \\ UNIVERSITY OF TOKYO \\ UNIVERSITY OF UTAH \\ WASHINGTON STATE UNIVERSITY \\ UNIVERSITY OF WASHINGTON \\ AMERICAN MATHEMATICAL SOCIETY
}

The Supporting Institutions listed above contribute to the cost of publication of this Journal, but they are not owners or publishers and have no responsibility for its contents or policies.

Mathematical papers intended for publication in the Pacific Journal of Mathematics should be in typed form or offset-reproduced (not dittoed), double spaced with large margins. Underline Greek letters in red, German in green, and script in blue. The first paragraph or two must be capable of being used separately as a synopsis of the entire paper. Items of the bibliography should not be cited there unless absolutely necessary, in which case they must be identified by author and Journal, rather than by item number. Manuscripts, in duplicate, may be sent to any one of the four editors. Please classify according to the scheme of Math. Reviews, Index to Vol. 39. All other communications should be addressed to the managing editor, or Elaine Barth, University of California, Los Angeles, California, 90024.

100 reprints are provided free for each article, only if page charges have been substantially paid. Additional copies may be obtained at cost in multiples of 50 .

The Pacific Journal of Mathematics is issued monthly as of January 1966. Regular subscription rate: $\$ 72.00$ a year (6 Vols., 12 issues). Special rate: $\$ 36.00$ a year to individual members of supporting institutions.

Subscriptions, orders for back numbers, and changes of address should be sent to Pacific Journal of Mathematics, 103 Highland Boulevard, Berkeley, California, 94708.

PUBLISHED BY PACIFIC JOURNAL OF MATHEMATICS, A NON-PROFIT CORPORATION

Printed at Jerusalem Academic Press, POB 2390, Jerusalem, Israel.

Copyright (C) 1977 Pacific Journal of Mathematics All Rights Reserved 


\section{Pacific Journal of Mathematics \\ Vol. 70, No. $2 \quad$ October, 1977}

B. Arazi, A generalization of the Chinese remainder theorem ........... 289

Thomas E. Armstrong, Polyhedrality of infinite dimensional cubes .... . . . 297

Yoav Benyamini, Mary Ellen Rudin and Michael L. Wage, Continuous

images of weakly compact subsets of Banach spaces ............ 309

John Thomas Burns, Curvature functions on Lorentz 2-manifolds ......... 325

Dennis F. De Riggi and Nelson Groh Markley, Shear distality and equicontinuity .................................. 337

Claes Fernström, Rational approximation and the growth of analytic

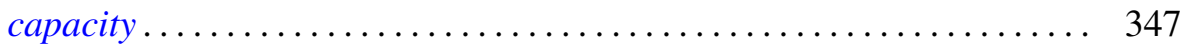

Pál Fischer, On some new generalizations of Shannon's inequality....... 351

Che-Kao Fong, Quasi-affine transforms of subnormal operators ......... 361

Stanley P. Gudder and W. Scruggs, Unbounded representations of

*-algebras........................................ 369

Chen F. King, A note on Drazin inverses .................... 383

Ronald Fred Levy, Countable spaces without points of first countability . . . 391

Eva Lowen-Colebunders, Completeness properties for convergence

spaces ......................................... 401

Calvin Cooper Moore, Square integrable primary representations ....... 413

Stanisław G. Mrówka and Jung-Hsien Tsai, On preservation of

E-compactness ................................ 429

Yoshiomi Nakagami, Essential spectrum $\Gamma(\beta)$ of a dual action on a von

Neumann algebra ................................ 437

L. Alayne Parson, Normal congruence subgroups of the Hecke groups

$G\left(2^{(1 / 2)}\right)$ and $G\left(3^{(1 / 2)}\right)$...

Louis Jackson Ratliff, Jr., On the prime divisors of zero in form rings . . . . 489

Caroline Series, Ergodic actions of product groups .................. 519

Robert O. Stanton, Infinite decomposition bases..................... 549

David A. Stegenga, Sums of invariant subspaces .................. 567 